BMC

Genomics

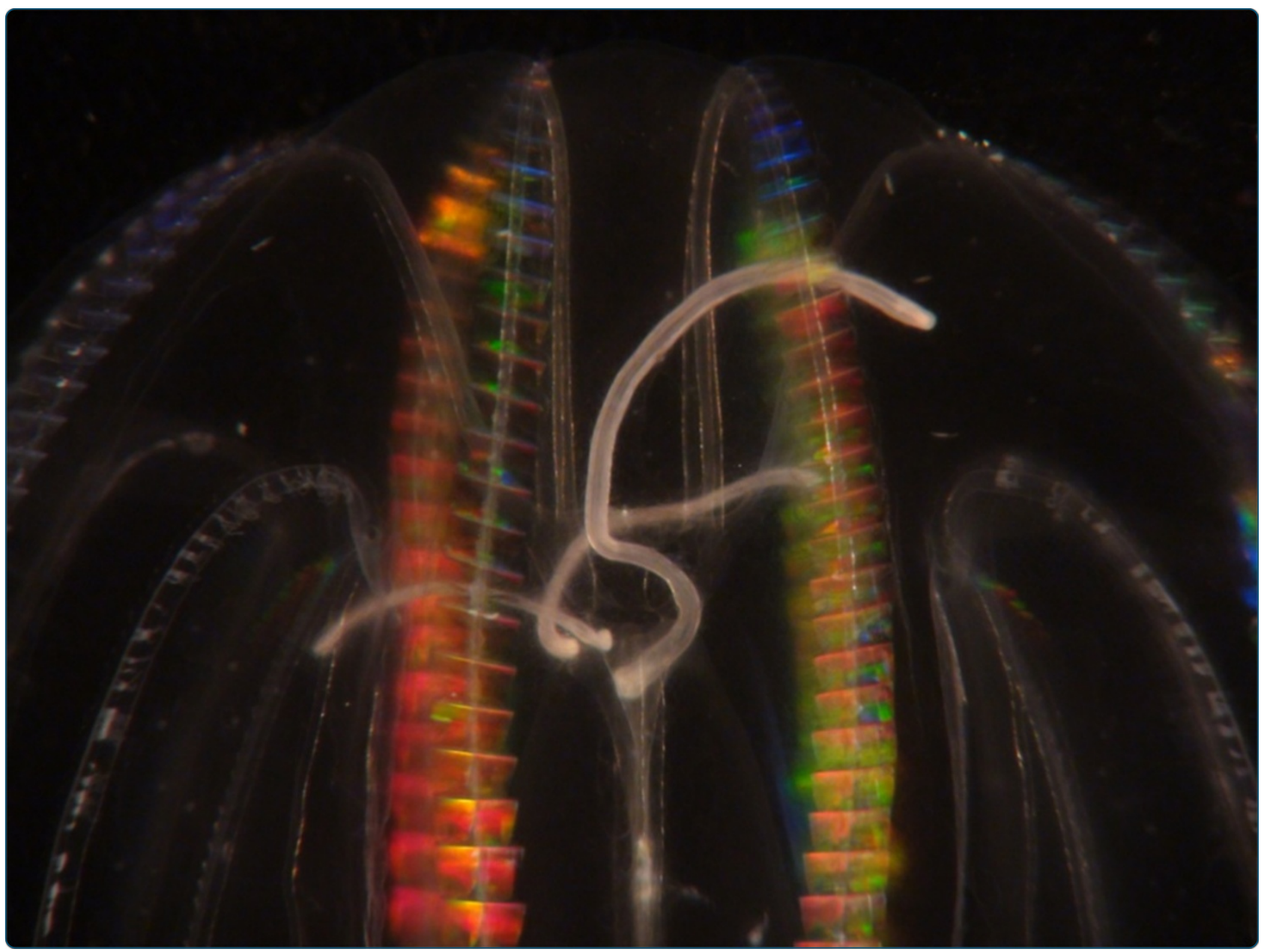

Production of a reference transcriptome and transcriptomic database (EdwardsiellaBase) for the lined sea anemone, Edwardsiella lineata, a parasitic cnidarian

Stefanik et al.

C Biomed Central 


\title{
Production of a reference transcriptome and transcriptomic database (EdwardsiellaBase) for the lined sea anemone, Edwardsiella lineata, a parasitic cnidarian
}

Derek J Stefanik ${ }^{1 \dagger}$, Tristan J Lubinski ${ }^{1 \dagger}$, Brian R Granger ${ }^{1,2+}$, Allyson L Byrd² ${ }^{2}$ Adam M Reitzel $^{3}$, Lukas DeFilippo ${ }^{4}$, Allison Lorenc ${ }^{4}$ and John R Finnerty ${ }^{1,2,4^{*}}$

\begin{abstract}
Background: The lined sea anemone Edwardsiella lineata is an informative model system for evolutionary-developmental studies of parasitism. In this species, it is possible to compare alternate developmental pathways leading from a larva to either a free-living polyp or a vermiform parasite that inhabits the mesoglea of a ctenophore host. Additionally, E. lineata is confamilial with the model cnidarian Nematostella vectensis, providing an opportunity for comparative genomic, molecular and organismal studies.

Description: We generated a reference transcriptome for E. lineata via high-throughput sequencing of RNA isolated from five developmental stages (parasite; parasite-to-larva transition; larva; larva-to-adult transition; adult). The transcriptome comprises 90,440 contigs assembled from >15 billion nucleotides of DNA sequence. Using a molecular clock approach, we estimated the divergence between E. lineata and N. vectensis at 215-364 million years ago. Based on gene ontology and metabolic pathway analyses and gene family surveys (bHLH-PAS, deiodinases, Fox genes, LIM homeodomains, minicollagens, nuclear receptors, Sox genes, and Wnts), the transcriptome of E. lineata is comparable in depth and completeness to N. vectensis. Analyses of protein motifs and revealed extensive conservation between the proteins of these two edwardsiid anemones, although we show the NF-KB protein of $E$. lineata reflects the ancestral structure, while the NF-KB protein of N. vectensis has undergone a split that separates the DNA-binding domain from the inhibitory domain. All contigs have been deposited in a public database (EdwardsiellaBase), where they may be searched according to contig ID, gene ontology, protein family motif (Pfam), enzyme commission number, and BLAST. The alignment of the raw reads to the contigs can also be visualized via JBrowse.

Conclusions: The transcriptomic data and database described here provide a platform for studying the evolutionary developmental genomics of a derived parasitic life cycle. In addition, these data from E. lineata will aid in the interpretation of evolutionary novelties in gene sequence or structure that have been reported for the model cnidarian $N$. vectensis (e.g., the split NF-KB locus). Finally, we include custom computational tools to facilitate the annotation of a transcriptome based on high-throughput sequencing data obtained from a "non-model system."
\end{abstract}

\footnotetext{
* Correspondence: jrf3@bu.edu

${ }^{\dagger}$ Equal contributors

'Department of Biology, Boston University, 5 Cummington Mall, Boston, MA 02215, USA

2Bioinformatics Program, Boston University, 24 Cummington Mall, Boston, MA 02215, USA

Full list of author information is available at the end of the article
} 


\section{Background}

Parasitism is arguably the dominant trophic strategy on earth, as the number of parasitic species is thought to exceed the number of free-living species, perhaps by 4-to-1 or more [1,2]. Presumably, every cellular organism is subject to parasitism, and parasites affect their hosts in a number of profound ways. For instance, parasites have helped to drive the evolution of sex [3-5] and immune systems [6]. They can markedly change the behavior of their hosts [7], influence host species' mating strategies and genetic variation $[8,9]$, and contribute to the decline of locally threatened populations $[10,11]$. However, despite the prevalence of parasitism and its clear ecological and evolutionary importance, parasitic species are relatively poorly characterized. For example, of the 1.5 million species currently named by taxonomists, less than $1 \%$ are known to be parasites $[12,13]$.

The evolution of parasitism from an ancestral free-living state can be accompanied by radical alterations to an organism's ontogeny, bodyplan, and life history (e.g., polyembryony in parasitoid wasps; [14]). Despite this, relatively few studies have explored the developmental evolution of parasitism, mainly because there are practical and theoretical hurdles to such studies. Foremost, it is often difficult to culture parasites in a laboratory setting, as maintaining an obligate parasite requires co-culture of a suitable host. Furthermore, in long-established obligate parasites, the initial steps in their developmental evolution are often obscured by their lengthy evolutionary divergence from free-living outgroups. Finally, parasites are generally not regarded as "model" systems, since parasitic life cycles are often highly derived and therefore not representative of the ancestral free-living condition in major organismal lineages. However, it has been argued that parasites should be of particular interest to evolutionary-developmental biology precisely because their tight associations with host species create "highly integrated reproductivedevelopmental-ecological systems" that are persistent through space and time [15].

The lined sea anemone, Edwardsiella lineata, does not exhibit the practical and theoretical limitations that hinder the study of many other parasites, making it a good model for evolutionary developmental studies of parasitism. The larva of E. lineata (Figure 1A) parasitizes the pelagic ctenophore Mnemiopsis leidyi (Figure 1B; [16]). More than 50\% of $M$. leidyi have been found to harbor parasites at Woods Hole, MA, which falls within the native range of the ctenophore [17]. In the North Sea, where the ctenophore has been introduced, up to $6.3 \%$ of individuals were found to harbor the parasite [18]. E. lineata can enter its host through the body wall or the mouth, eventually coming to reside adjacent to the stomach or one of the eight radial canals that exit the stomach $[16,17,19]$. When situated in the host, E. lineata assumes a novel vermiform body plan
(Figure 1C; [20]) and feeds upon the ctenophore's gut contents. When ready to exit the host, or upon death of the host, the parasite morphs from the elongated form into a planula larva. The planulae are active swimmers, and can follow one of two developmental trajectories, depending on the environment: if presented with a new host, they can reassume the vermiform body plan of the parasite; however, in the absence of a second host, they can settle and develop into an adult polyp [17]. The polyps live in dense aggregates on the seafloor or on other available hard substrates. Importantly, the parasite is easily collected in infected ctenophores, and it can be maintained indefinitely in the lab as an adult polyp or for several weeks as a parasite inside a ctenophore host (Stefanik, unpublished data). The derived developmental trajectory that leads from the planula to the vermiform parasite can be compared directly to the ancestral anthozoan developmental pathway that leads from the planula to the polyp. Additionally, the ontogeny of E. lineata may be compared to that of the starlet sea anemone, Nematostella vectensis, which is a leading cnidarian model system for development and genomics [21-24] and a member of the same family as E. lineata (Edwardsiidae) [25].

To inform our knowledge of the E. lineata gene repertoire, and how changes in expression of particular genes may contribute to ontogenetic changes associated with a derived life history, we sequenced and assembled the transcriptome of E. lineata from developmental stage-specific cDNA libraries. We created a database, EdwardsiellaBase, as a platform to share sequence information from $E$. lineata and facilitate queries of gene expression across developmental stages. Both the raw reads and assembled transcriptomic sequences are publicly accessible via the web interface of EdwardsiellaBase.

\section{Construction and content}

\section{Sequencing and assembly}

Sequencing yielded $\sim 188.1$ million read pairs that passed Illumina's GAIIx quality filter (each read pair consisted of two $\sim 40$ nucleotide reads from the same original RNA transcript). The overall sequencing yield of this study ( 15,000 MB) exceeded that of all but two published cnidarian transcriptome sequencing projects (Figure 2). The reads were assembled using Velvet [26] and Oases [27] over a range of kmer values (21-39 nucleotides). The assembly comprises 90,440 contigs with an N50 of 1,036 basepairs.

To evaluate whether our sequencing effort provided thorough coverage of the libraries we constructed, we produced a saturation curve that relates the number of sequencing reads to the percentage of the 90,440 contigs covered by those reads (Figure 3 ). We began to reach saturation at around 250 million sequencing reads. Three replicates of randomly chosen samples of 250 million 


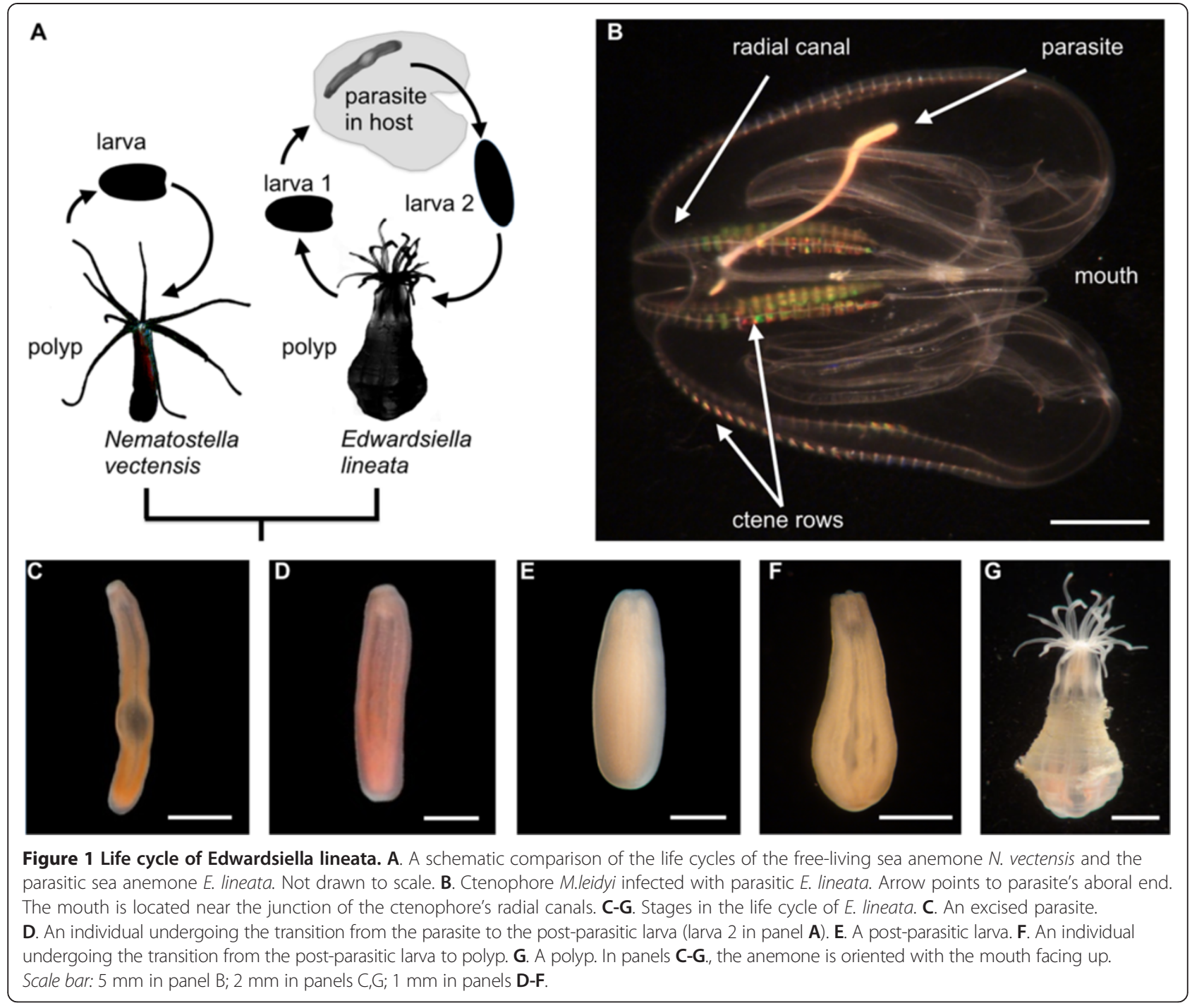

reads amounted to 10X coverage of $98.4 \%$ of the 90,440 contigs.

\section{Relationship to edwardsiid type specimens}

From our transcriptome assembly, we recovered a complete ribosomal RNA transcription unit (18S-ITS1-5.8SITS2-28S). We aligned the $18 \mathrm{~S}$ portion to previously published $18 \mathrm{~S}$ genes of six species from the family Edwardsiidae and one outgroup taxon (Metridium senile; Additional file 1). The edwardsiid data included three previously published $18 \mathrm{~S}$ sequences from $E$. lineata itself. Maximum likelihood analysis places the sequence obtained in this study in a clade of four E. lineata sequences, with bootstrap support of $81 \%$ (Figure 4).

\section{Molecular divergence dating}

We used a molecular clock approach based on seven concatenated protein-coding genes [37] to estimate the divergence date between $E$. lineata and $N$. vectensis. The analysis included seven cnidarians in addition to 81 noncnidarian taxa for which the full complement of protein sequences is available and robust estimates of divergence times from the fossil record exist ([37-47]; Additional file 2). $N$. vectensis appears as the most closely related taxon to E. lineata in the analysis (Figure 5). The divergence time between these two edwardsiid anemones was estimated between 215-364 million years. This compares to an estimated divergence time of 504-652 million years between sea anemones (Actinaria) and hard corals (Scleractinia), which is consistent with a recently published report [28].

\section{Taxonomic affinity and inferred phylogenetic antiquity of sequences}

Of the 90,440 contigs in our transcriptome assembly, 40\% $(36,234)$ produced BLAST hits to sequences in NCBI's non-redundant (NR) protein database, while $60 \%(54,206)$ had no BLAST hits (Figure 6A). Most of the raw reads 


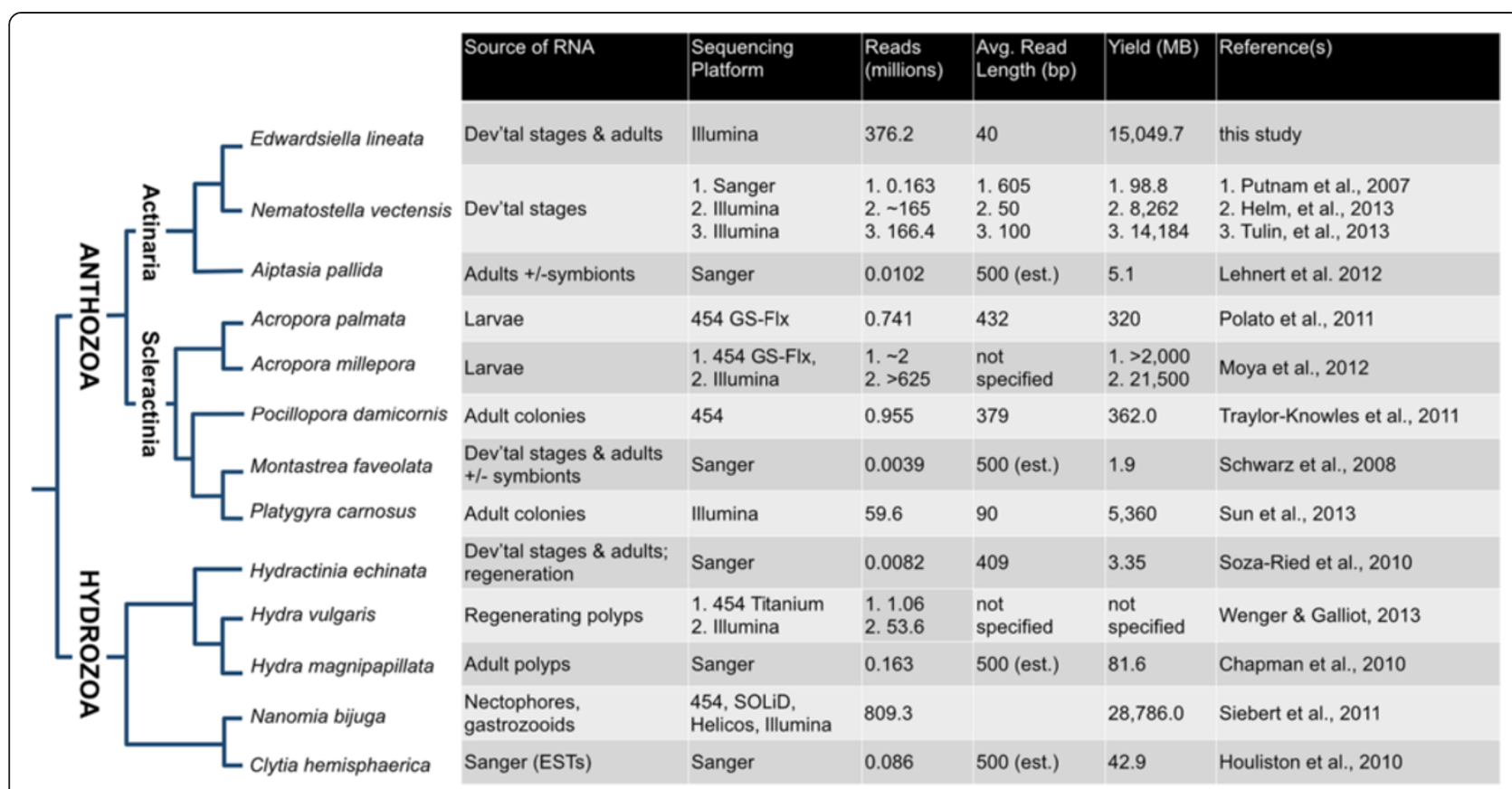

Figure 2 Published transcriptome sequences for cnidarians. The methodology and sequencing yield for published cnidarian transcriptomes are summarized here. Taxa are arranged based on their phylogenetic relationships, as compiled from [25,28-32].

(>71\%) map to those contigs that produce BLAST hits (Additional file 3). Ninety-one percent of the contigs that fail to produce BLAST hits are short (100-500 nucleotides in length; Additional file 4). Nearly three-quarters of the contigs that produced a BLAST hit (73.5\%) had a top hit to $N$. vectensis (Figure 6B).

Taxonomically restricted BLAST searches were performed so that we could provisionally ascribe the origin of each of the E. lineata transcripts to a particular evolutionary ancestor. For example, a transcript shared with other animal lineages but not non-metazoan eukaryotes or prokaryotes would be assigned to the metazoan ancestor. Using this approach, we infer that $19.2 \%$ of the genes producing BLAST hits originated in the common ancestor of Eubacteria and Eukaryotes, another 10.8\% originated in the common ancestor of animals, and $2.2 \%$ originated in the common ancestor of cnidarians (Figure 7). Sequences producing hits to distantly related lineages, but not to more closely related lineages (e.g., to "Eubacteria" but not to "Bilateria," "basal Metazoa," or "other Eukaryota") probably represent contaminating organisms. Approximately $16 \%$ of genes that produced BLAST hits matched only to sequences from the other edwardsiid anemone, $N$. vectensis.

\section{Gene ontology (GO) analysis}

Of the approximately $40 \%$ of contigs $(36,234)$ that produced a BLAST hit to a protein sequence in the non-redundant database at NCBI, roughly half $(18,613)$ could be associated

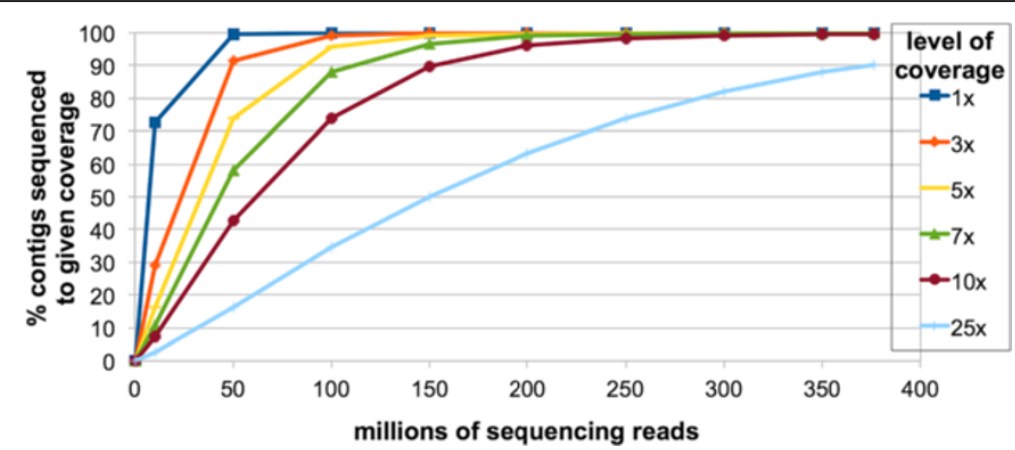

Figure 3 Sequencing saturation curve. The percentage of contigs with nominal coverage of $n$-fold ( $Y$-axis) is plotted against the number of sequencing reads (X-axis). Sequencing sub-samples of a given size were randomly selected from the total pool of sequencing reads. Three replicates were performed for each data point. The mean value is shown. The standard error was too small to represent visually on this graph. 


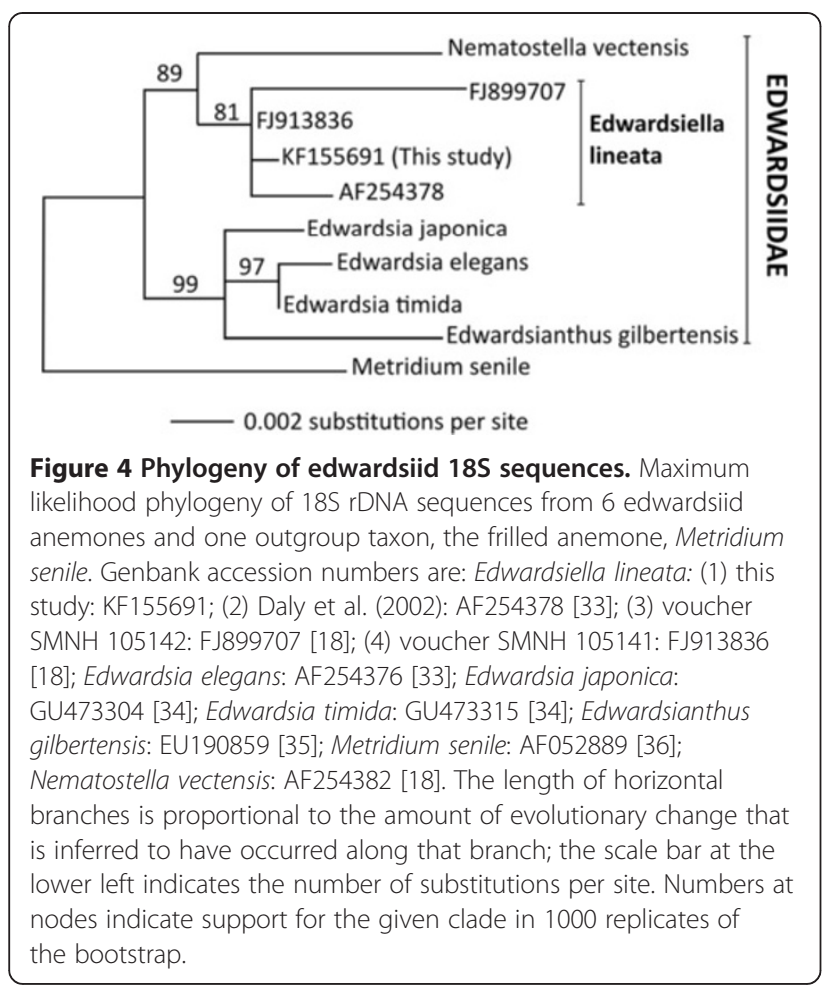

with one or more $\mathrm{GO}$ annotation terms. In total, these contigs matched 4,246 GO terms. Because most contigs match many GO terms, there are a total of 244,321 pairings between contigs and GO terms. Using an in-house script (Additional file 5), we tallied the number of matches to the GO terms in the most inclusive subcategories under "Molecular Function" (Figure 8), "Biological Process" and "Cellular Component" (Additional file 6). To place these results in context, we performed the same GO analysis on published ESTs from $N$. vectensis [49]. In general, there was a close correspondence between the recovery of particular GO categories in these two edwardsiid sea anemones. In the 20 categories under "Molecular Function" where a match was possible, we recovered a match from one or both of the anemones for $17 \mathrm{GO}$ categories. For these 17 $\mathrm{GO}$ categories, we retrieved an equal number of hits for both anemones in three instances, a slightly greater number of hits for $N$. vectensis in three instances, and a greater number of hits for $E$. lineata in 11 instances. With respect to Molecular Function (Figure 8), the recovery of a greater number of hits in $E$. lineata versus $N$. vectensis was most pronounced for "negative regulation of molecular function" (15 for E. lineata vs. 10 for N. vectensis), "receptor activity" (21 vs. 12), and "enzyme regulator activity" (19 vs. 13).

\section{Metabolic pathway analysis}

To identify metabolic pathways represented by the assembled contigs, we extracted the Enzyme Commission (EC) numbers from our Blast2GO results for E. lineata. We then cross-referenced these with EC numbers already assigned by The Kyoto Encyclopedia of Genes and Genomes (KEGG; [50]) to predicted genes in N. vectensis. Overall, there are 5935 EC numbers, of which, 638 are associated with $N$. vectensis. One or more EC numbers could be associated with 2,148 of the E. lineata contigs. These contigs produced matches to 594 EC numbers, of which, 408 are shared between $N$. vectensis and $E$. lineata, while 186 were found in $E$. lineata but not $N$. vectensis. The metabolic pathways represented by the $E$. lineata contigs and $N$. vectensis predicted genes were diagrammed using iPath 2.0 (Figure 9; Additional file 7; [51]).

\section{Recovery of specific genes and gene families from $E$. lineata} To evaluate the comprehensiveness of this transcriptome, we searched for E. lineata representatives of eight different gene families that have already been surveyed in $N$. vectensis [52-59]: bHLH-PAS, deiodinases, Fox genes, LIM homeodomains, minicollagens, nuclear receptors, Sox genes, and Wnts. We also sought to identify the transcription factor NF- $\mathrm{kB}$ among the $E$. lineata contigs, because a number of functional studies have been performed on NF-KB in $N$. vectensis [60-63], and the overall structure of the protein in this species [64] appears to be derived relative to the ancestral condition for metazoans [65]. To identify members of these gene families in E. lineata, we used the known $N$. vectensis sequences to query the E. lineata transcriptome using reciprocal BLAST searches (see Methods). Our

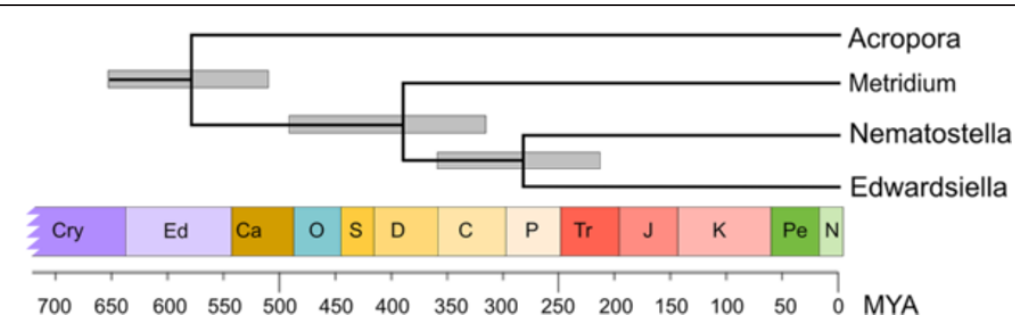

Figure 5 Estimation of the Nematostella-Edwardsiella divergence. A portion of a Bayesian phylogenetic tree, based on seven concatenated protein-coding genes and three ribosomal DNAs [37,48], used to date the divergence between Nematostella and Edwardsiella. The complete analysis comprises 87 taxa (see Methods), but the tree has been pruned so that only the anthozoan clade (corals and sea anemones) is shown. The thick gray bars at each internal node represent the $95 \%$ confidence interval for the given divergence time. 


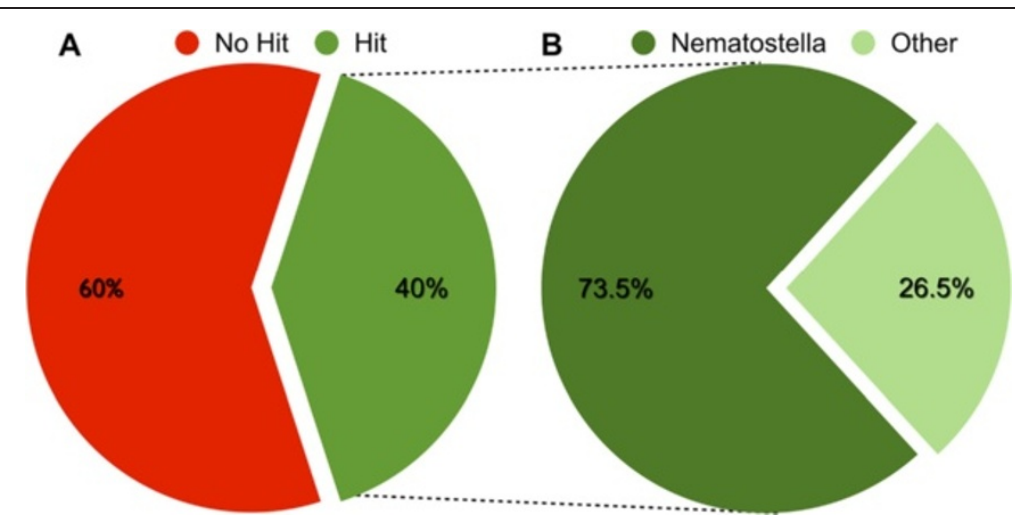

Figure 6 Summary of BLAST hits. A. All 90,440 contigs in the assembly were compared to sequences in NCBI's non-redundant protein database using BLASTX, and $40 \%$ produced one or more matches to sequences in the database at a threshold Expect value of -3 . B. Of the $40 \%$ percent of contigs producing BLAST hits, $73.5 \%$ had a top hit to a sequence from $N$. vectensis.

searches recovered an equivalent or nearly equivalent number of gene family members in E. lineata as had been previously reported for $N$. vectensis (Table 1 ).

To evaluate the phylogenetic relationships among gene family members, we performed maximum likelihood analyses for bHLH-PAS, deiodinases, LIM homeodomains, minicollagens, nuclear receptors and Wnts. With the exception of minicollagens, each gene family analysis was based on protein sequences from deuterostome (human) and cnidarian ( $N$. vectensis; E. lineata) lineages. Minicollagens are specific to cnidarians, and therefore the minicollagen tree contains no deuterostome sequences. A phylogeny of Wnt genes is presented in Figure 10 (all other phylogenetic trees are contained in Additional file 8). Based on the phylogenetic analyses, in almost all cases, for each previously reported $N$. vectensis gene, we recovered an E. lineata ortholog. For example, in the Wnt phylogeny, both anemones possess representatives of 12 out of 13 Wnt subfamilies, and within each of these subfamilies, the sister-group to a sequence from $N$. vectensis is a sequence from E. lineata. The only Wnt subfamily not represented in E. lineata or $N$. vectensis is Wnt9. The protein motif analysis (Figure 10) revealed extensive conservation among Wnt proteins from humans and edwardsiid anemones. Of

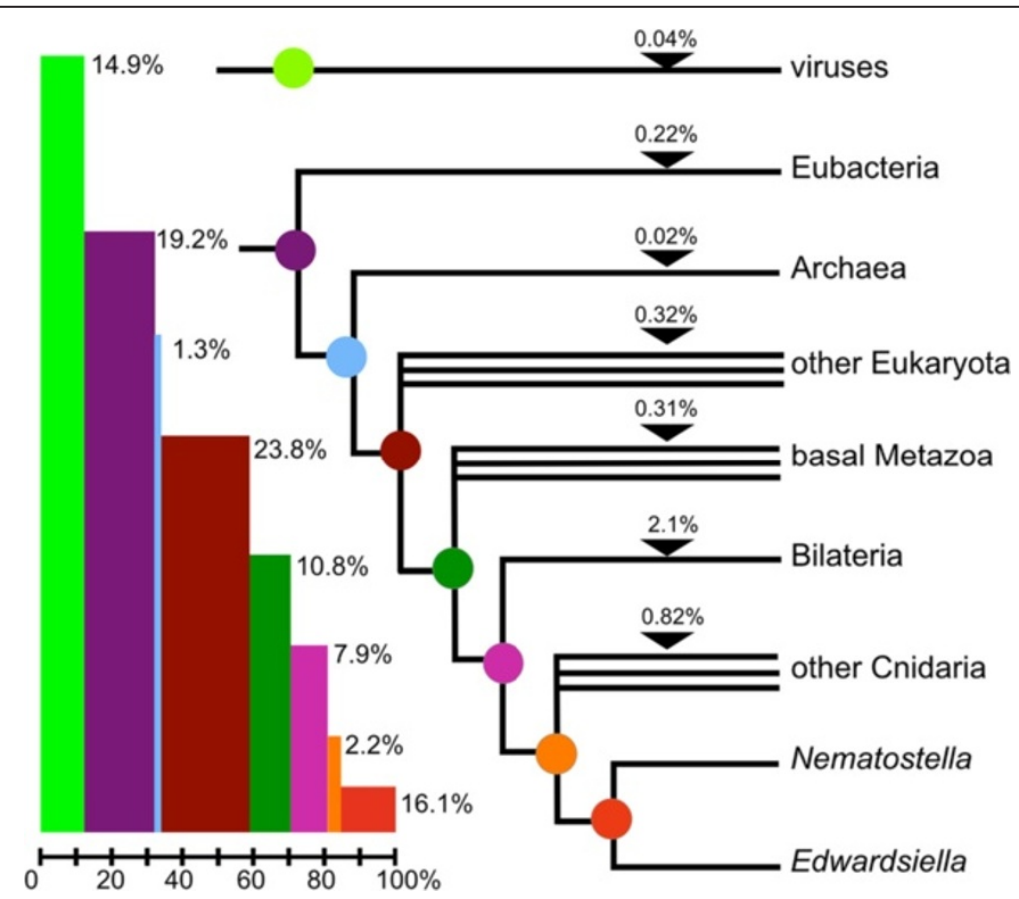

Figure $\mathbf{7}$ Inferred phylogenetic antiquity of $\mathbf{E}$. lineata genes. On the basis of phylogenetically nested BLAST searches, each E. lineata contig was tentatively assigned to a particular branch of the phylogeny shown here. 


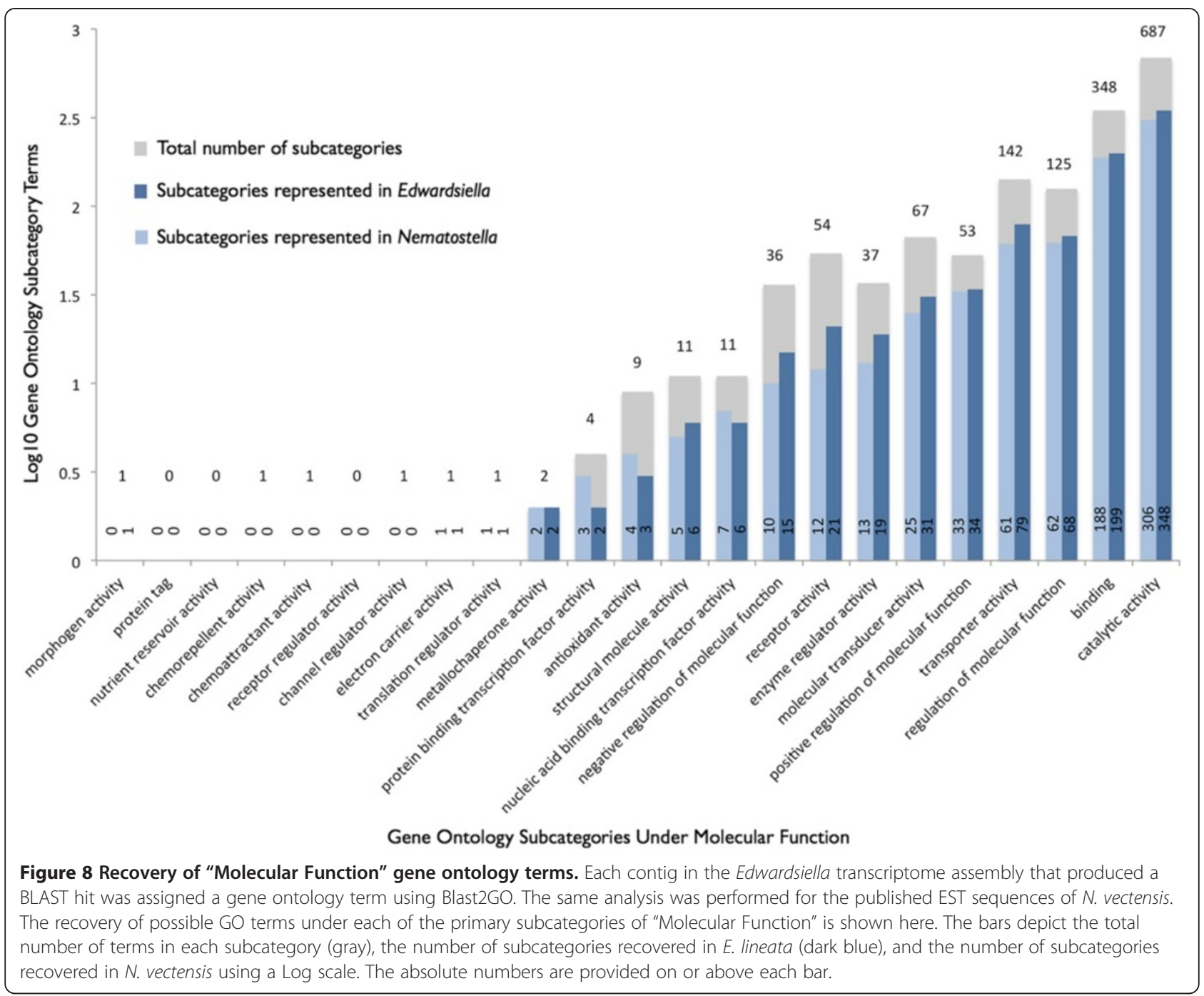

note, all but three of the E. lineata Wnt transcripts (the exceptions being Wnt10, Wnt6, and Wnt7B) encode predicted proteins that share all motifs found in their $N$. vectensis orthologs.

As previously reported for $N$. vectensis [66], we have identified two Wnt7 splice variants in E. lineata. In the Wnt phylogeny (Figure 10), the two N. vectensis variants (7A and 7A) appear most closely related to each other, as do the two E. lineata variants (7A and $7 \mathrm{~B})$. This is due to the fact that, within each species, the splice variants share a substantial amount of sequence identity (Figure 11). However, the $N$. vectensis Wnt7A appears to share the same exon composition with E. lineata Wnt7A, while the $N$. vectensis Wnt7B shares the same exon structure with $E$. lineata Wnt7B. A phylogenetic analysis of all four sequences based upon only the regions of the protein they share in common groups $N$. vectensis Wnt7A with $E$. lineata Wnt7A and $N$. vectensis Wnt7B with $E$. lineata Wnt7B (Figure 11).
As in N. vectensis [64], there appears to be only one $\mathrm{NF}-\kappa \mathrm{B}$ family member in E. lineata. However, unlike $N$. vectensis, the single $E$. lineata $\mathrm{NF}-\mathrm{kB}$ reflects the ancestral structure in that it contains both an $\mathrm{N}$-terminal Rel Homology Domain (RHD) and a C-terminal inhibitory IкB domain consisting of multiple ankyrin repeats (Figure 12). In $N$. vectensis, the ancestral NF- $\mathrm{kB}$ locus is split, so that the RHD and I $\mathrm{B}$ domains are encoded by separate loci $[64,65]$.

\section{Utility}

The raw sequencing reads and the contigs generated from our transcriptome assembly are housed at EdwardsiellaBase (http://www.EdwardsiellaBase.org), whose overall organization is based on PocilloporaBase [67]. The database was populated as follows (Figure 13; blue arrows). Each of the assembled contigs is associated with a Contig ID, nucleotide sequence, and sequence length. Those contigs that produced a BLAST hit at NCBI are also associated with 

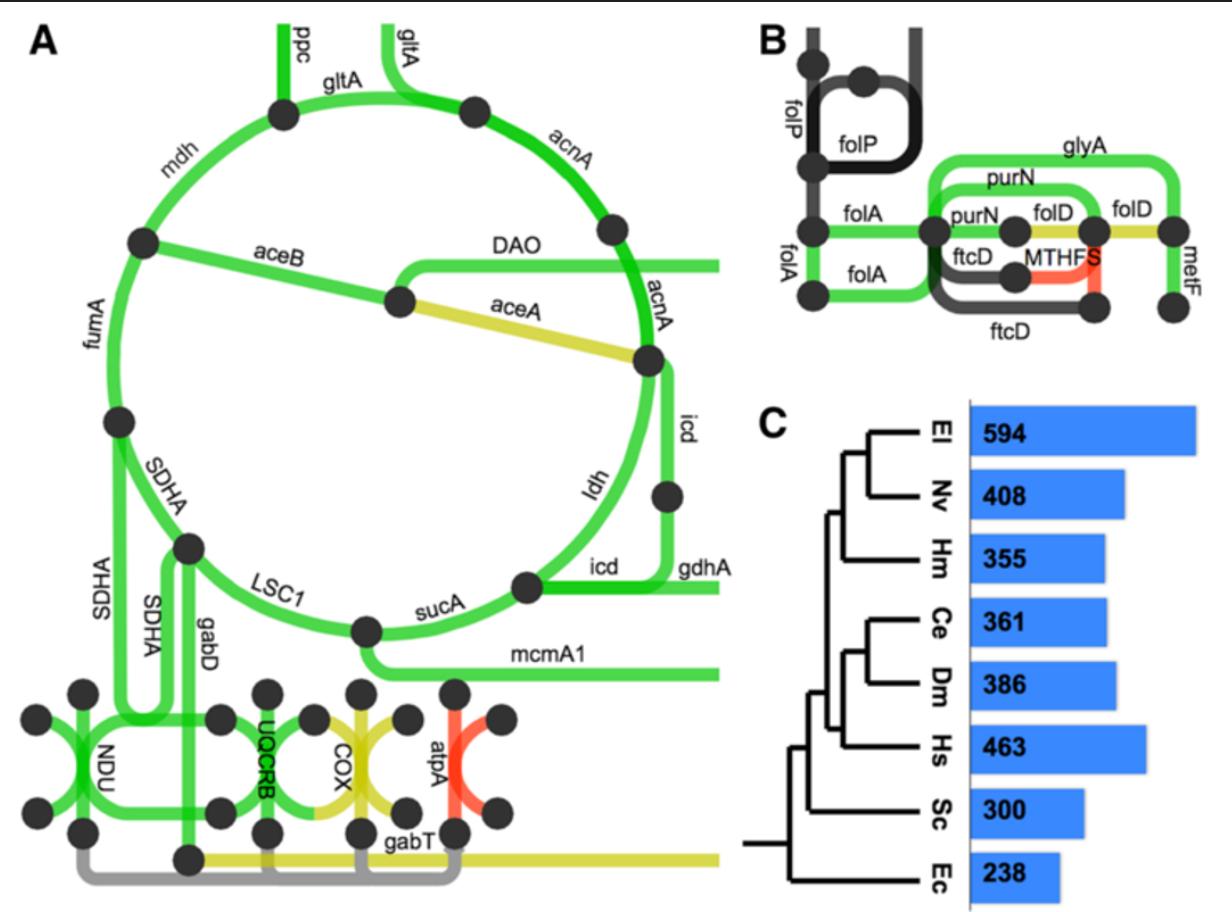

Figure 9 Recovery of metabolic pathway components. The networks shown above depict $\mathbf{A}$. the Krebs cycle, and $\mathbf{B}$. the folate pathway as represented by iPath. The nodes represent metabolites, and the edges represent metabolic transformations. Green edges indicate pathways that were found in both $N$. vectensis and $E$. lineata. Red pathways were only found in N. vectensis, and yellow pathways were only found in $E$. lineata. Gray and black edges indicate pathways that were not found in either anemone, in the case of gray edges because no Enzyme Commission numbers map to these edges, and thus they were impossible to detect in our analysis. List of gene name abbreviations for panels $\mathrm{A}$ and $\mathrm{C}$ are as follow: glt $\mathrm{A}=$ citrate synthase; $\mathrm{mdh}=$ malate dehydrogenase; $\mathrm{ace} B=$ malate synthase $\mathrm{A} ; \mathrm{DAO}=\mathrm{D}$-amino-acid oxidase; ace $\mathrm{A}=$ isocitrate lyase; gdh $\mathrm{A}=$ glutamate dehydrogenase; suCA = 2-oxoglutarate decarboxylase; $\mathrm{LSC1}=$ succinate-CoA ligase; $\mathrm{mcmA1}=$ methylmalonyl-CoA mutase $\mathrm{N}$-terminal domain; $\mathrm{SDHA}=$ succinate dehydrogenase complex, subunit A; gabD = succinate-semialdehyde dehydrogenase $\mathrm{l}$; UQCRB = ubiquinol-cytochrome $\mathrm{c}$ reductase binding protein; folP = dihydropteroate synthase; glyA = serine hydroxymethyltransferase; purN = phosphoribosylglycinamide formyltransferase; metF = 5,10-methylenetetrahydrofolate reductase; $\mathrm{MTHFS}=5,10$-methenyltetrahydrofolate synthetase; $\mathrm{ftcD}$ = glutamate formiminotransferase; fol $\mathrm{A}=$ dihydrofolate reductase; fol $\mathrm{D}=$ bifunctional 5,10-methylene-tetrahydrofolate dehydrogenase; $\mathrm{ppc}=$ phosphoenolpyruvate carboxylase; acnA = aconitate hydratase 1; icd - isocitrate dehydrogenase; Idh = L-lactate dehydrogenase; fumA = fumarate hydratase; COX = Cytochrome $\mathrm{C}$ oxidase; atpA = ATP synthase subunit alpha; gabT = 4-aminobutyrate aminotransferase; NDU = NADH dehydrogenase. $\mathrm{C}$. A selection of species that share ancestry with $E$. lineata at various evolutionary distance. The bar graph and numbers represent the amount of shared EC numbers between that species and Edwardsiella lineata. The species are E. coli, S. cereviseae, H. sapiens, D. melanogaster, C. elegans, H. magnipapillata, N. vectensis, and E. lineata.

Table 1 Recovery of gene family members from Edwardsiella

\begin{tabular}{ccccc}
\hline & & \multicolumn{2}{c}{$\boldsymbol{N}$. vectensis } & \\
\cline { 3 - 4 } Gene family & E. lineata & Published studies $^{\mathbf{1}}$ & ESTs $^{\mathbf{2}}$ & Human \\
\hline bHLH-PAS & 7 & $7[48]$ & 7 & 11 \\
Deiodinase & 5 & $4[49]$ & 1 & 3 \\
Fox & 17 & $14[50]$ & 16 & 42 \\
LIM homeodomain & 6 & $6[44,45]$ & 4 & 12 \\
Minicollagens & 3 & $5[47]$ & 5 & - \\
Nuclear receptors & 10 & $17[46]$ & 12 & 48 \\
Sox & 12 & $14[50]$ & 9 & 20 \\
WNT & 13 & $12[43]$ & 7 & 19 \\
\hline
\end{tabular}

${ }^{1}$ Published studies on individual gene families; ${ }^{2}$ Expressed sequence tags generated as part of the Nematostella genome sequencing project [49]. the protein accession numbers from the top five hits, and these numbers were used to retrieve additional information from NCBI (Gene/Protein Name and Species Name/Taxon ID). Then, using Blast2GO, the protein accession numbers were used to retrieve information about biochemical pathways (Enzyme Commission Number; Enzyme Name) and gene ontology (Gene Ontology ID; Gene Ontology Term). All contigs were translated in all six frames and searched using HMMer to identify conserved protein domains (Pfam Accession Number; Pfam Motif Name; Pfam Description Keyword). The raw reads were aligned to the assembled contigs using Bowtie 2 (v. 2.0.0-beta; [68]).

The data can be searched by Contig, Protein Family, Metabolic Pathway or Gene Ontology (Figure 13; red arrows). EdwardsiellaBase also supports the complete range of BLAST options to search the assembled contigs for matches to a query sequence. Finally, the JBrowse $[69,70]$ 


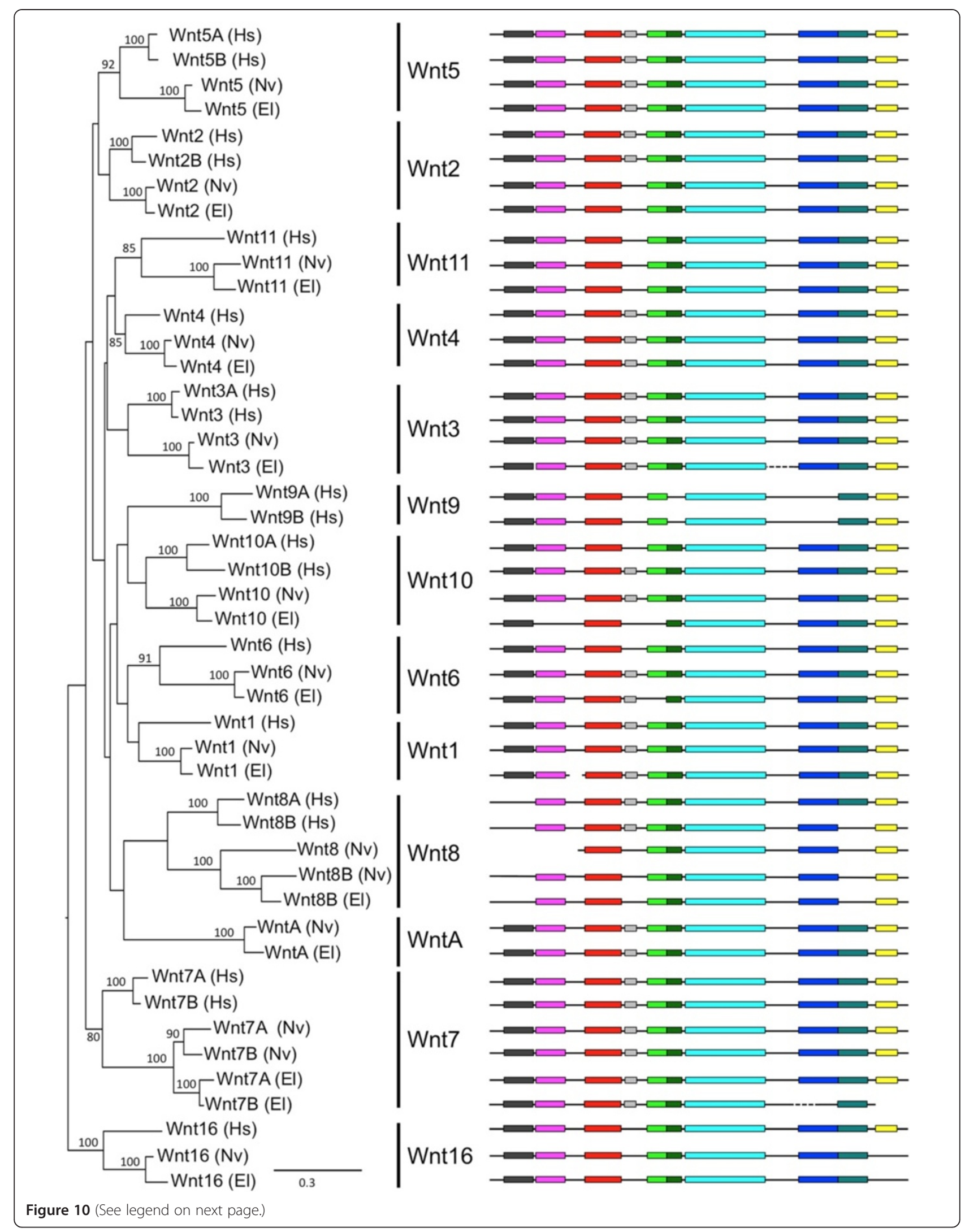


(See figure on previous page.)

Figure 10 Maximum likelihood tree of Wnt genes. The tree shown is based on a maximum likelihood analysis of an amino acid alignment of the Wnt consensus motif (PF00110). Numbers at nodes represent bootstrap values above 80\%. Branch length is shown in terms of expected number of substitutions per residue (bar at lower right). Conserved motifs were identified using MEME, as described in the methods. Motifs (colored boxes) are drawn to scale, but the inter-motif regions (black lines) were altered to allow the motifs to align for ease of visualizing conservation in motif composition and order.

function enables one to view alignments of the raw reads to the assembled transcriptome to help assess validity of transcripts. A literature database allows users to search the published literature on Edwardsiella using matches to keywords or any user-entered text string. The database structure and entity relationships are depicted in Additional file 9.

\section{Discussion}

\section{Evidence that the transcriptome is representative}

The present study describes a transcriptome assembly for $E$. lineata based on roughly 15 billion nucleotides of RNA sequencing. This is one of the largest transcriptomic datasets currently available for any cnidarian [28,67,71-85], and approximately 2.5 times the sequencing yield estimated to be sufficient for assembling a representative transcriptome [86]. To ensure that we captured transcripts expressed throughout E. lineata's complex life cycle, we generated cDNA libraries from five distinct developmental stages. Our saturation analysis showed that (Figure 3) additional sequencing of these libraries would result in identification of relatively few novel transcripts. Evidence that the transcriptome assembly is representative of the expressed gene repertoire of an edwardsiid anemone is the comparable recovery of GO terms (Figure 8; Additional file 6) and gene families (Table 1) (Figures 10, 11 and 12; Additional file 6) from E. lineata and N. vectensis. Taken together, these data suggests that our sequencing effort was sufficient to produce a representative transcriptome that captures a large fraction of the transcript variety encoded by the E. lineata genome. Undoubtedly, we have failed to capture some transcripts that are expressed at
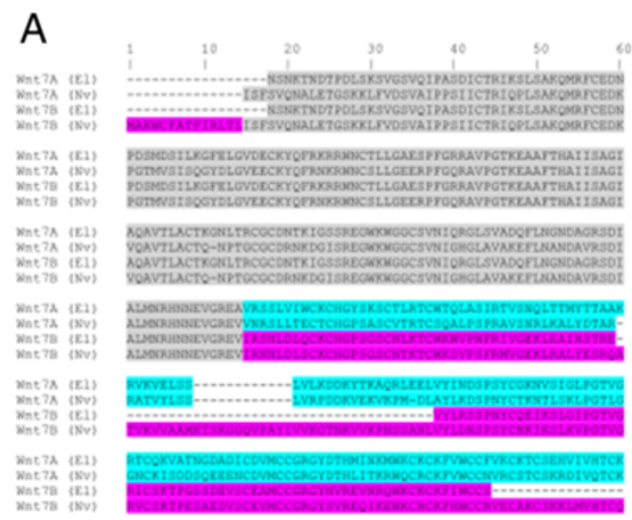

B
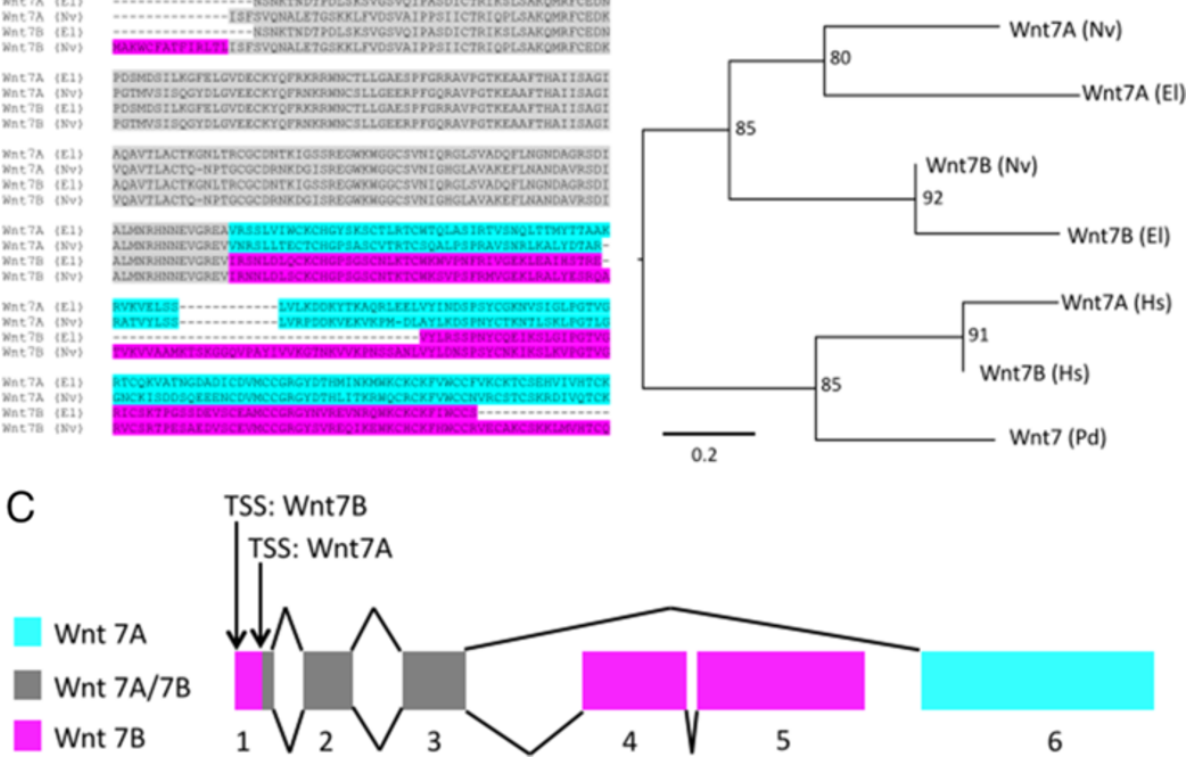

TSS: Wnt7B
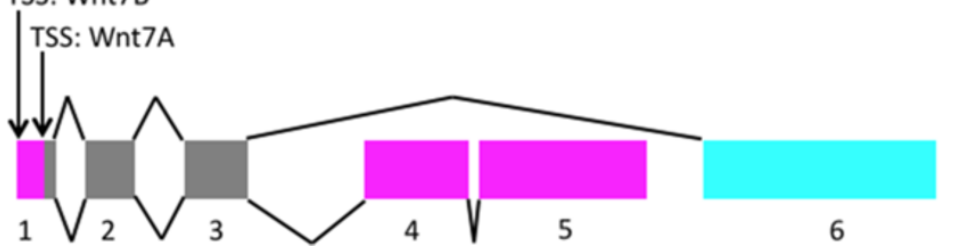

Figure 11 Wnt7 splice variants in Edwardsiella and Nematostella. A. Amino acid alignment of Wnt7A and 7B transcripts from E. lineata and $N$. vectensis. In the gray region, the amino acid sequence and the underlying nucleotide sequence of $E$. lineata Wnt7A is identical to that of $E$. lineata Wnt7B. Similarly, the amino acid sequence and underlying nucleotide of $N$. vectensis Wnt7A is identical to that of $N$. vectensis Wnt7B. In the regions of the alignment highlighted in blue and pink, the amino acid sequence of $E$. lineata Wnt7A is most similar to $N$. vectensis Wnt7A (blue) and the amino acid sequence of $E$. lineata Wnt7B is most similar to N. vectensis Wnt7B. B. A maximum likelihood phylogeny based on amino acid sequences of Wnt7A and 7B but excluding the portion of the alignment shared by $E$. lineata Wnt7A and Wnt7B (the region shaded in gray). Numbers at nodes indicate how many times the given clade was recovered in 1000 replications of the bootstrap. The scale bar represents the number of substitutions per site. Taxon abbreviations are as follows: $\mathrm{El}=\mathrm{Edwardsiella} \mathrm{lineata;} \mathrm{Hs}=$ Homo sapiens; Nv $=$ Nematostella vectensis. C. Diagram of the Nematostella Wnt7 locus illustrating the similarities and differences of the Wnt7A/7B splice variants (adapted from [66]). Wnt7A is composed of sequences from exons $1 \mathrm{~b}, 2,3$, and 6 , and Wnt7B is composed of exons $1,1 \mathrm{~b}, 2,3,4$, and 5 . 


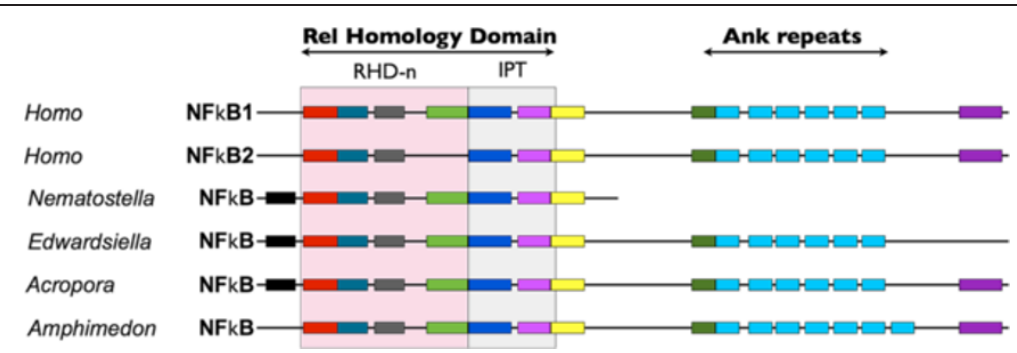

Figure 12 Conservation and loss of motifs in NF-KB proteins. Conserved protein motifs were identified using MEME. Motifs (colored boxes) are drawn to scale, but the inter-motif regions (black lines) were altered to allow the motifs to align for ease of visualizing conservation in motif composition and order. The sequences included in the analysis were the NF-kB proteins of three cnidarians (Acropora millepora, E. lineata, N. vectensis) and one sponge (Amphimedon queenslandica) as well as the NF-KB1 and NF-KB2 proteins of Homo sapiens.

very low levels during the developmental stages studied here, or that are expressed only in different developmental, physiological, or environmental contexts.

\section{Utility of E. lineata for comparative transcriptomics} and genomics

The utility of any species for comparative transcriptomic and genomic studies depends on its relationship to other taxa for which extensive sequence data are available. Molecular, morphological, and developmental characters support the placement of E. lineata within the family Edwardsiidae and the subfamily Milneedwardsiinae, a clade comprising the genera Edwardsiella, Nematostella, Drillactis, and Paraedwardsia [25,33,87]. The $18 \mathrm{~S}$ phylogenetic analysis performed here confirms the specimens we characterized as E. lineata. This confirmation is important,

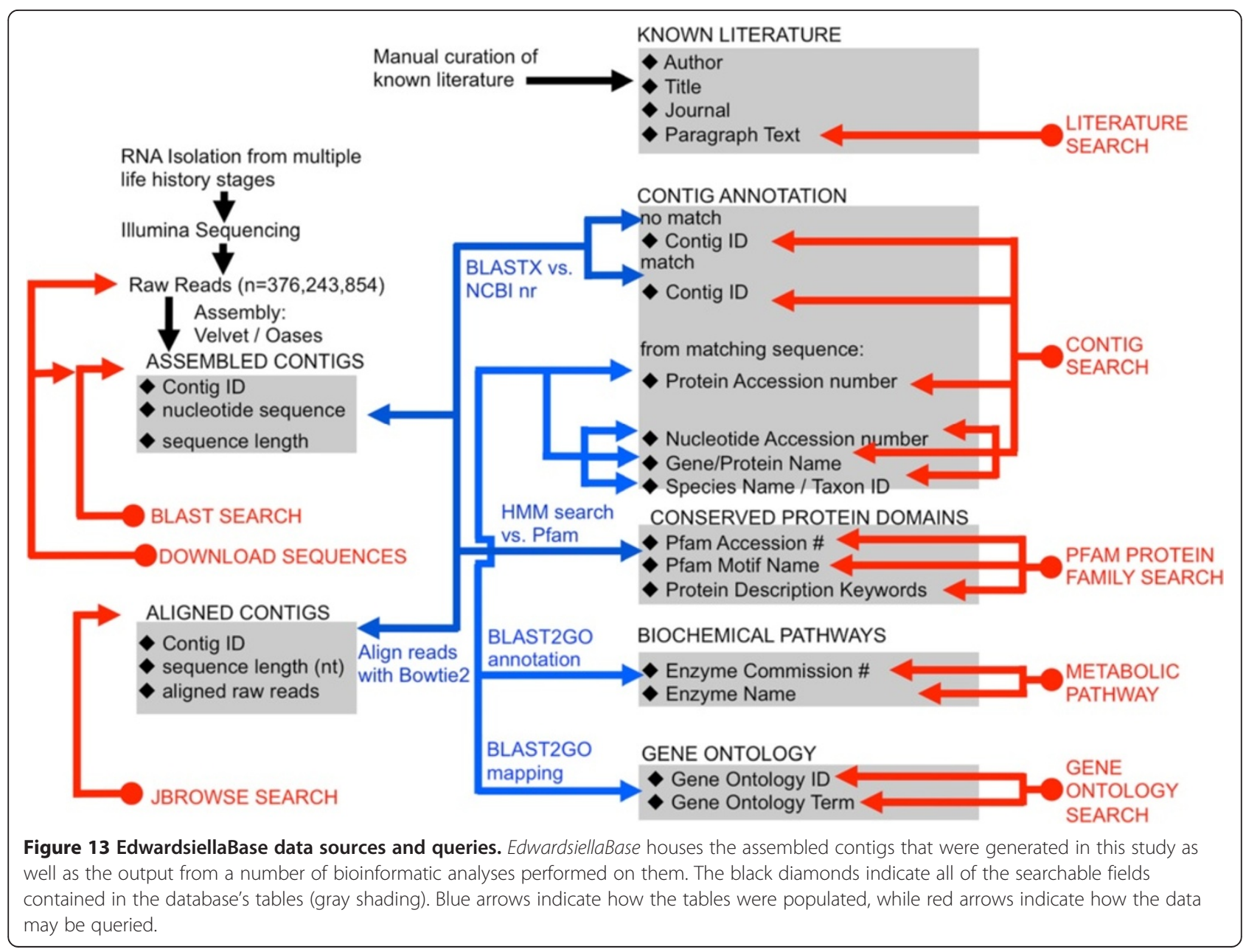


given that we are seeking to establish a reference transcriptome for the species. The $18 \mathrm{~S}$ phylogenetic analysis also supports the placement of Edwardsiella and Nematostella within the Milneedwardsiinae (Figure 4). Thus, this study supports the conclusion that E. lineata is one of the closest living relatives of $N$. vectensis. Our molecular clock estimate (Figure 5) suggests the divergence between Nematostella and Edwardsiella occurred sometime between the early Triassic Period (215 mya) and the early Devonian (>360 mya). As $N$. vectensis protein-coding genes appear to evolve at a rate comparable to, or even slower than vertebrates [49], the evolutionary distance between Edwardsiella and Nematostella is likely sufficient to facilitate the identification of functional conservation in protein sequence and structure; i.e., at this distance, sequence conservation is not likely to reflect mere phylogenetic inertia. Looking forward, comparing genome sequences between these two edwardsiid anemones is likely to be useful in identifying conserved cis-regulatory sequences, as has been done for echinoderm species spanning divergences from 35-500 mya [88,89].

\section{BLAST based annotation}

Forty percent of the assembled contigs in the E. lineata transcriptome produced BLAST hits to sequences in NCBI's non-redundant (NR) protein database, while 60\% did not match any protein sequences in the database (Figure 6). This ratio between BLAST hits and misses for contigs within the E. lineata transcriptome is comparable to another published cnidarian transcriptome assembly for the coral Pocillopora damicornis [67]. The high percentage of contigs in the E. lineata assembly that do not produce BLAST hits may be a function of contig size. Ninety-one percent of the contigs that fail to produce BLAST hits are relatively short (100-500 nucleotides in length; Additional file 4). Since BLAST scores are influenced by sequence match length, shorter sequences will produce lower scores, and may also be more likely to represent assembly artifacts or truncated transcript models. Over two-thirds of the raw reads $(>71 \%)$ map to contigs that produce BLAST hits (Additional file 3 ).

Another explanation for the presence of contigs in the $E$. lineata transcriptome assembly that produced no BLAST hits to NR protein database is that some of the contigs may represent assembly of long, non-coding RNA transcripts, for which no cognate protein would exist in the NR database. We used BLASTn to query the NONCODE database [90] with the set of contigs that produced no hits against the NR protein database. This search yielded matches for 354 contigs. The $E$. lineata transcriptome assembly therefore contains non-coding transcripts, but these transcripts represent a small fraction of the total contigs that produced no BLAST hits to the NR protein database.

Given the key position of cnidarians in metazoan phylogeny - as the likely sister group to triploblastic bilaterians - there is widespread interest in pinpointing the evolutionary origin of cnidarian genes. For example, which genes have been conserved since the time of the eumetazoan common ancestor, and which genes are cnidarian inventions? We approached this question using taxonomically restricted BLAST searches (Figure 7). Using this approach, we can ascribe putative origins to the genes that encode the $E$. lineata transcripts we recovered. For example, $19.2 \%$ of the E. lineata contigs generated significant matches to sequences from other Eukaryota, plus Eubacteria, and Archaea, suggesting (1) that these genes originated prior to the origin of Eukaryota, and (2) they have been conserved in eukaryotes and prokaryotes since that time. The number of hits produced from this analysis can be influenced by a few confounding factors, which should be considered when viewing the results. While it is possible that these sequences represent shared transcripts of essential function common to the organisms to which we ascribed their origin and their descendent lineages; a potentially confounding variable is that it is also possible that some of these sequences are transcripts produced by other organisms residing within and/or on the focal taxon, and which were subsequently sequenced and deposited in the $\mathrm{nr}$ database, or represent unintended taxonomic sampling from the holobiont of the anemone in this study. Due to potentially confounding factors, and the relatively permissive BLAST cutoff threshold utilized, the analysis of taxonomic affinity in this study represents a provisional phylogenetic stratigraphy of gene origins. To achieve a more robust assignment of origin across the taxonomic breadth of this study, one would need to produce multiple sequence alignment and phylogenetic trees for each of the 90,440 transcripts in the E. lineata transcriptome.

The BLAST-based approach used here is currently limited by the uneven representation of major taxonomic groups in the NCBI database, including the phylum Cnidaria. While over $16 \%$ of the E. lineata sequences generated significant matches to $N$. vectensis alone, only $2.2 \%$ generated matches to other cnidarians in addition to Nematostella. This disparity is likely a reflection of the relatively large amount of data from $N$. vectensis in the database. As more cnidarian taxa are sequenced, we expect many of the sequences from E. lineata that currently generate hits to Nematostella alone will be shared across the phylum.

\section{Gene ontology (GO) analysis}

We were able to assign 17 GO subcategory terms under the "Molecular Function" ontology to transcripts from either the $N$. vectensis ESTs and E. lineata sequencing produced from this study (Figure 8). Sixteen of these subcategories were represented in transcripts from both sea anemones. However, the Molecular Function subcategory 
of "morphogen activity" was only assigned to sequences from E. lineata. Of the remaining 16 subcategories, there is a generally close correspondence in presence/absence of subcategories within each ontology between the expressed sequence resources from each sea anemone. Taken together, these findings suggest that the transcriptome assembly produced for E. lineata is comparably representative of the expressed transcript repertoire of an edwardsiid sea anemone as the $N$. vectensis ESTs. This interpretation is based on the assumption that these two confamilial sea anemones would exhibit similar gene ontology distributions in their expressed transcripts as a function of shared, derived physiological and genomic characteristics.

\section{Recovery of selected gene families in E. lineata}

The largely consistent recovery of orthologous genes from seven divergent gene families in E. lineata and $N$. vectensis suggests that the genetic repertoire of these two edwardsiid anemones is well conserved and that the reference assembly described here provides thorough coverage of the E. lineata transcriptome. Figure 10 depicts a Maximum Likelihood phylogenetic analysis of Wnt sequences from E. lineata, N. vectensis, and human, alongside a MEME analysis of the protein coding domains of these transcripts. This analysis reveals extensively conserved protein motif architecture across Wnt proteins between cnidarians and human (the deuterostome representative). Additionally, motif conservation is high between the two sea anemones, with the entire motif complement for each protein being conserved between $N$. vectensis and E. lineata, with the exception of three transcripts (Wnt10, Wnt6, and Wnt7B) in which one or more motifs are discordant between the two taxa. All E. lineata sequences used in this analysis represent single contigs (with the exception of Wnt3, Wnt1 and Wnt7B, which were conceptually spliced). Taken together with the degree of protein coding motif conservation between the two sea anemones, this suggests that many contigs represent full-length transcripts. The detailed analysis of Wnt7 sequences (Figure 11) also clearly supports the conclusion that the Wnt7A/7B splice variants are conserved between $N$. vectensis and E. lineata.

\section{No evidence for pervasive change in the gene repertoire of this parasite}

This study has produced no evidence for pervasive changes in the gene repertoire of E. lineata that might have evolved in concert with the evolution of its novel parasitic life cycle. In contrast, a recent study on four cestodes identified extensive losses of genes and pathways that are broadly conserved in other animals as well as the origin of specialized metabolic pathways adapted to extract nutrients from the host [91]. This is to be expected given that cestodes are an ancient lineage of obligate internal parasites. Although we cannot date the antiquity of parasitism in $E$. lineata, except to say that it must postdate the last common ancestor with $N$. vectensis, we should not expect extensive gene losses in E. lineata, as this parasitic anemone retains all of the life cycle stages present in related free-living anemones. Therefore, it would presumably require the same developmental regulatory genes and metabolic pathways. Despite its derived life cycle, we expect that there will be genes and proteins for which $E$. lineata reflects the primitive condition, while the free-living $N$. vectensis, an important cnidarian model system, exhibits a derived condition. NF- $\mathrm{BB}$ is such an example, as the NF- $\mathrm{KB}$ protein of E. lineata reflects the ancestral protein structure, in which the DNA-binding domain and inhibitory domain are contained within the same transcript, whereas these domains are split between two separate loci in N. vectensis (Figure 12). As an interesting aside, NF- $\mathrm{KB}$ appears to be one of the genes lost in parasitic cestodes [91]. We expect that E. lineata has evolved some genetic modifications that would make it better able to exploit its host ctenophore, though these may be few in number. A detailed analysis of differential gene expression between developmental stages, which is beyond the scope of this paper, is currently underway.

\section{Functionality of EdwardsiellaBase}

EdwardsiellaBase was modeled after the previously published species-specific cnidarian databases PocilloporaBase [67], and StellaBase [92,93], but it expands upon their functionality in key ways. As with these published databases, an html-based interface allows users to search the assembled contigs using contig identifiers, enzyme names or EC numbers, protein families (Pfam), protein names, and Gene Ontology (GO) information (Figure 13). The database also features a fully equipped BLAST interface for searching the assembled contigs based on sequence similarity to known genes and proteins. New functions include a literature search, JBrowse alignment viewer [69,70], and individual contig pages. The literature search allows the user to query the E. lineata literature, much of which has been published in relatively inaccessible venues, such as books that are out of print. The JBrowse feature allows users to view alignments of reads to assembled contigs to and visualize the relative abundance of transcripts, including alternate splice forms. The individual contig page summarizes available information, and also provides a notes section, to which users can submit entries. Provisional gene names have been assigned to each contig that produced a BLAST hit using Blast2GO. The database may be searched using these gene names, and when a name has been assigned to a given contig, that name is provided on the contig information page.

It is also possible to search for matches to a query sequence using the complete set of BLAST options. BLAST 
searches return a standard BLAST page, with a few additional features.

\section{Conclusions}

We describe the sequencing and assembly of a reference transcriptome for the parasitic cnidarian, the lined sea anemone, E. lineata. This dataset represents a significant contribution to the comparative study of cnidarian transcriptomes because of (1) the overall sequencing yield ( 15,000 Mb of nucleotide sequence), (2) the phylogenetic placement of $E$. lineata as the closest cnidarian taxa to $N$. vectensis for which appreciable molecular sequence data exist, and (3) the fact that E. lineata is a recently evolved parasite whose novel life cycle is tractable to laboratory investigation. The assembled transcripts published in this study capture the large majority of the transcriptome of this sea anemone. The diversity of Gene Ontology terms, metabolic pathways components, and gene family members we were able to recover from the E. lineata contigs compares favorably with published EST data from $N$. vectensis. The assembled contigs are available in a searchable database, EdwardsiellaBase, that will serve as a platform for studying the evolutionary developmental genomics of $E$. lineata's novel, derived parasitic life history, and will be useful for comparative transcriptomic studies between cnidarian taxa, particularly between $E$. lineata and $N$. vectensis. The scripts and computational tools employed in this study are included in the supplementary files to facilitate the annotation of transcriptome assemblies from other emerging model systems for which genomic data are not available.

\section{Availability and requirements}

EdwardsiellaBase is freely available at http://edwardsiellabase.org.

\section{Methods}

Animal collection and developmental sampling

Ctenophores (Mnemiopsis leidyi) infected with E. lineata were collected from July through October of 2009 and 2010 at Woods Hole, MA as previously described [17]. Approximately two-hundred $E$. lineata parasites were extracted from approximately $70 \mathrm{M}$. leidyi using forceps and a scalpel. Approximately 30 of these excised parasites (Figure 1C) were immediately harvested for RNA isolation. The remaining parasites were transferred to full-strength artificial seawater (Instant Ocean; salinity $=36$ parts per thousand) and maintained at room temperature, so they could continue their development [17]. Individuals were then selected to represent particular developmental stages based on the duration of their incubation and their gross morphological appearance. To represent the parasiteto-planula transition stage (Figure 1D), approximately 30 of the developing anemones were collected for RNA isolation
12-24 $\mathrm{h}$ after their excision from the host. The anemones at this stage of development exhibited the following three phenotypic and/or behavioral criteria: (1) reduction in pharynx length relative to the parasitic stage, (2) ability to move via cilia, and (3) an overall body shape that was intermediate between the vermiform parasite and the ovoid planula. To represent the larval stage (the planula; Figure 1E), approximately 30 anemones were allowed to develop for 2-4 days post host excision. The planulae exhibited the following characteristics: (1) lack of transparency, (2) vigorous swimming ability, and (3) ovoid shape. Thirty of the remaining larvae were allowed to develop until they began showing signs of metamorphosis into polyps (Figure 1F), such as (1) cessation of swimming and (2) tentacle eruption. The adult stage (Figure 1G) was represented by individuals that successfully metamorphosed into polyps capable of using their tentacles to feed on freshly hatched brine shrimp larvae (Artemia salina). Six individuals were harvested for RNA isolation at this stage.

\section{RNA isolation, library preparation and sequencing}

Total RNA was isolated from pooled specimens for each of the five developmental stages (Figure 1C-G). For the four pre-adult stages (parasites, the parasite-to-larva transition, larvae, and the larva-to-polyp transition), we used $\sim 30$ individuals in each case, which is equivalent to $\sim 100 \mathrm{mg}$ of tissue. For the adult polyp we isolated RNA from 6 individuals. For the pre-adult stages, total RNA was isolated using TRIzol (Life Technologies) according to the manufacturers protocol. From adult polyps, total RNA was isolated using the Omega Biotek Mollusk RNA Isolation Kit. Subsequently, mRNA was isolated from each pool of total RNA using the Poly(A) Purist mRNA isolation kit (Ambion). Separate cDNA libraries were prepared for each of the five developmental stages using the mRNA Sample Preparation Kit from Illumina. Sequencing of cDNA libraries was performed on a Genome Analyzer IIx (Illumina). Each library was sequenced on an individual lane of a flow cell using 40-bp, paired-end reads. Overall, the five libraries yielded a total of $376,243,854$ sequencing reads that passed the Illumina GAIIx quality filter.

\section{Assembly}

Each stage-specific library was individually assembled using Velvet (version 1.1.05; [26]) and Oases (version 0.1.22; [27]). For the adult, we used a kmer range of 25-39; for all other stages we used a kmer range of 21-39. For all other assembly parameters, we used the default settings for Velvet and Oases. The individual assemblies were then merged using both Velvet and Oases to produce a single reference transcriptome. The merged assemblies comprise 90,440 contigs. 


\section{Assessment of sequencing coverage}

We used a random re-sampling approach to assess how sequencing depth affected recovery of transcripts. All reads from all stages were aligned to the reference file using Bowtie 2 (v. 2.0.0-beta; [68]). The resulting sam file was then parsed with a custom python script (Additional file 10) that randomly selects a given number of reads from the total reads without replacement. This script then returns a file listing the nominal coverage of all contigs, based on the contig length, read length, and number of reads aligned to each contig. The file can then be easily parsed to assess the amount of contigs above each coverage threshold. We evaluated subsets of the total reads ranging from 0 to all of the reads in increments of 50 million. The analysis was performed 3 times for each subset size, except for the 0 and "all" read sets, as the replicates of these sets are guaranteed to be exactly the same each time. For each data point, the standard deviation was calculated, and found to be negligible (all less than $0.1 \%$ of the total contigs that pass a given coverage threshold).

\section{Divergence date estimation}

To estimate the divergence between $E$. lineata and $N$. vectensis, we used a molecular clock approach based on the published multi-gene alignment of Erwin et al. [48]. This alignment comprises seven nuclear housekeeping genes (aldolase, methionine adenosyltransferase, ATP synthase beta chain, catalase, elongation factor 1 alpha, triosephosphate isomerase and phosphofructokinase; [37]) and three ribosomal DNAs (5.5S, 18S, and 28S rDNA) from 119 taxa. We restricted our analysis to taxa for which Erwin et al. [48] included fossil calibration points (Additional file 2). The resulting alignment included 87 taxa (Additional file 11). We used BLAST searches to identify orthologs of all these genes from $E$. lineata. The $E$. lineata sequences were manually added to the alignment.

The alignment of protein coding and ribosomal genes was input into MrBayes (version 3.1.2 [94], as implemented in the CIPRES Science Gateway, version 3.3), and a phylogeny was estimated using mixed models for the protein and nucleotide partitions of the alignment. We set up one run of four chains using two unlinked GTR + gamma models: an amino acid GTR + gamma model was applied to the amino acid partition, and a nucleotide GTR + gamma model was applied to the rDNA partition. The shape of the gamma distribution was estimated using four rate categories for each partition. Chains were allowed to run for $1,000,000$ generations, with a burn-in of $25 \%$, and sampling every 5,000. The resulting tree for the full set of 87 taxa can be viewed in Additional file 2.

Bayesian estimation of divergence dating was carried out using the program Phylobayes (version 3.3b; $[95,96]$ ). The current iteration of Phylobayes does not support mixed (protein and nucleotide) datasets for divergence dating, so we followed the example established by Erwin et al. [48] and used just the protein-coding characters for the divergence dating analysis. The chronogram resulting from Phylobayes is available in Additional file 2.

\section{Transcriptome annotation}

All 90,440 contigs were compared against the nonredundant (NR) database on NCBI using BLASTx at a threshold Expect value of 1E-03. Contigs with no match were BLASTed against a database of noncoding nucleotides on the NONCODE database [90] to search for homology to transcribed RNAs that are not translated into protein.

From the BLAST results, the taxonomic source of the top five hits obtained for each contig were stored in EdwardsiellaBase. To estimate the phylogenetic origin of sequences in the $E$. lineata transcriptome, protein lists were downloaded from NCBI using a series of scripts (Additional file 12) for a selection of taxonomic categories encompassing taxa of increasingly distant evolutionary relationship to E. lineata. The taxonomic categories used were: (1) $N$. vectensis, (2) Cnidaria excluding $N$. vectensis, (3) Bilateria, (4) Metazoa excluding Cnidaria and Bilateria, (5) Eukaryota excluding Metazoa, (6) Archaea, (7) Eubacteria, and (8) viruses. For this search, we also used BLASTx at a threshold Expect value of 1E-03.

$\mathrm{GO}$ terms were assigned to contigs through the Blast2GO servers after importing the BLAST results. Production of informative graphs about the GO data was generated through analysis of the data via a custom Python script (Additional file 5) which parses a file (gene_ontology.obo) from the Gene Ontology ftp site containing information about each node and its parent(s) and children. From this, information about the GO hierarchy is parsed by the script, and stored temporarily. Using the recovered GO data, and a starting node in the hierarchy, the script then looks for nodes below the starting node in the hierarchy for which GO data was recovered in the transcriptome data in order to determine the coverage of the sub-hierarchy. With this script, a user can identify all the contigs associated with a particular GO term and its subtree. In our analysis, we grouped all contigs according to the highest sub-category under the principal GO categories: Biological Process, Cellular Component, and Molecular Function (Figure 8; Additional file 6).

Blast2GO annotated contigs with Enzyme Commission (E.C.) numbers when applicable. Available E.C. numbers for $N$. vectensis were obtained through the Kyoto Encyclopedia of Genes and Genomes (KEGG; [50]). The E.C. numbers for E. lineata and N. vectensis were compared to see which enzymes were in both sets, and which were exclusive to one anemone or the other. Enzymes were then formatted, and cross-referenced to an edge list file from the interactive tree of life to produce a file (Additional file 13), which was 
uploaded to the iPath2.0 program for visualization (Figure 9; Additional file 7; [51]).

\section{Recovery of gene families from $E$. lineata}

We compiled FASTA files containing published protein sequences from $N$. vectensis for bHLH-PAS genes, deiodinases, Fox genes, LIM homeodomains, minicollagens, nuclear receptors, Sox genes, and Wnts. We then queried the $E$. lineata transcriptome with these sequences using tBLASTn. The top 10 hits from E. lineata were retained from each query. These were used to perform reciprocal BLASTx searches versus the FASTA file containing the protein sequences from $N$. vectensis to verify that each $E$. lineata sequence is most similar to the original query sequence. This sequence of BLAST searches was performed using a custom Python script (Additional file 14). In the case of all gene families except minicollagens (which are unique to Cnidaria), predicted protein sequences were obtained from $N$. vectensis, E. lineata, and Homo sapiens. Sequences were aligned using MUSCLE [97], and amino acid characters with gaps were removed from the alignment. The resulting gap-free alignments were then analyzed using ProtTest (v.3; [98]) to determine the best-fit model of amino acid replacement according to the Akaike Information Criterion. Maximum-likelihood phylogenies were estimated from the edited alignments using the default parameters of RaxML-HPC2 [99] as implemented at the CIPRES Science Gateway [100]. To evaluate the support for interior nodes, 1000 replicates of the bootstrap were performed [101]

A complete $18 \mathrm{~S}$ rDNA transcript was recovered from the specimens sequenced for this study via a BLAST search of EdwardsiellaBase using $N$. vectensis $18 \mathrm{~S}$ rDNA as a query sequence. This $18 \mathrm{~S}$ sequence was then aligned to published $18 \mathrm{~S}$ sequences for eight other edwardsiid anemones using the default parameters of MUSCLE [97]. Gaps and poorly-aligned regions were removed with Gblocks [102]. The edited alignment is available in Additional file 1. A maximum-likelihood phylogeny was estimated from this edited alignment using the default parameters of RaxMLHPC2 [99] as implemented at the CIPRES Science Gateway [100]. To evaluate the support for interior nodes, 1000 replicates of the bootstrap were performed [101].

For all protein families examined here, we used MEME (Multiple Expectation Maximization for Motif Elicitation; [103]) to identify conserved motifs in orthologs and paralogs from the various species sampled. Motif searches were performed under the following settings: maximum number of motifs $=10$; occurrences of a single motif $=$ any number; minimum length of a motif $=5$ amino acids; maximum length of a motif $=100$. Conserved motifs are depicted in the relevant figures to the right of each gene's name (Figures 10, 12; Additional file 8 ).

\section{Database construction}

EdwardsiellaBase is a relational database constructed in PostgreSQL (version 8.4.4). It houses the E. lineata contigs generated in this study in addition to the results from a number of bioinformatics analyses performed on these contigs. The database structure and entity relationships are depicted in Additional file 9. Files to construct the database were prepared and parsed from resulting data, and available data from NCBI, Expasy, and amiGO. Web pages are generated in real time using Python scripts that query the database through the pgdb module for Python. The BLAST suite of programs (v. 2.2.24+) is installed on the server, and is run with a query against specific BLAST-formatted databases using the subprocess module of Python. The raw sequencing reads were aligned to the assembled contigs and preloaded into a file structure that allows the user to quickly locate and display alignment to a contig of interest through JBrowse (v. 1.7.6; [69,70]).

\section{Additional files}

Additional file 1: Edwardsiidae_18S_alignment. A nexus file containing $18 \mathrm{~S}$ rDNA sequences from six species of edwardsiid anemones and one outgroup taxon, Metridium senile.

Additional file 2: DivergenceDating_trees-clock-calib_85taxa. A pd file containing a table of fossil dates used to calibrate the molecular clock, the phylogenetic tree of 85 taxa from MrBayes, and the chronogram from Phylobayes.

Additional file 3: ReadsMappingToContigsProducingBlastHits. Bar graphs depicting (A) the average number of sequencing reads and (B) the overall number of sequencing reads that map to contigs that produce BLAST hits versus those contigs that do not produce BLAST hits.

Additional file 4: LengthOfContigsProducingBlastHits. A histogram depicting the frequency of a range of contig lengths for contigs that produce BLAST hits versus contigs that do not produce BLAST hits.

Additional file 5: GeneOntologyExtractionScript. A custom python script that extracts gene ontology terms from a gene_ontology.obo file.

Additional file 6: GeneOntology. Two bar graphs depicting the recovery of possible $\mathrm{GO}$ terms under each of the primary subcategories of "Biological Process" and "Cellular Component". The bars depict the total number of terms in each subcategory (grey), the number of subcategories recovered in Edwardsiella (dark blue), and the number of subcategories recovered in Nematostella (light blue) using a Log scale. The absolute numbers are provided on or above each bar.

Additional file 7: CompleteMetabolicNetwork. The complete collection of metabolic pathways as represented by iPath. The nodes represent metabolites, and the edges represent metabolic transformations. Green edges indicate pathways that were found in both $N$. vectensis and E. lineata. Red pathways were only found in $N$. vectensis, and yellow pathways were only found in E. lineata. Gray and black edges indicate pathways that were not found in either anemone, in the case of gray edges because no Enzyme Commission numbers map to these edges, and thus they were impossible to detect in our analysis.

Additional file 8: MaximumLikelihoodGeneTrees. Maximum likelihood gene trees for bHLH-PAS, deiodinases, LIM homeodomains, minicollagens, and nuclear receptors. With the exception of minicollagens, each gene family analysis was based on protein sequences from deuterostome (human), protostome (Platynereis dumerilii), and cnidarian (Nematostella vectensis; Edwardsiella lineata) lineages. The location of conserved motifs for each protein sequence is also shown. Details of individual phylogenetic analyses are contained within the file. 
Additional file 9: EdwardsiellaBaseEntityRelationship. A graphic depicting the database structure and entity relationships of EdwardsiellaBase. Details are contained within the file.

Additional file 10: SequencingSaturationCurveScript. A custom python script that randomly selects a given number of reads from the total reads without replacement and determines the fraction of the overall assembly that passes a certain coverage threshold.

Additional file 11: DivergenceDating_Alignment_85taxa. A Phylip file containing an alignment of amino acid sequences from seven concatenated proteins from 85 different taxa.

Additional file 12: NCBITaxonRestrictedRetrieval. A collection of custom python scripts that perform iterative, taxonomically restricted BLAST searches against the sequences housed at NCBI in an attempt to infer the phylogenetic generality and evolutionary origin of gene sequences.

Additional file 13: iPathInput. An edge list file from the interactive tree of life that can be read by iPath2.0 to visualize the presence or absence of metabolic pathways in $E$. lineata and $N$. vectensis.

Additional file 14: GeneFamilyRecoveryScript. A script to detect gene families in transcriptome assemblies using biopython and BLAST. Inputs include a fasta file of protein sequences and fasta files for multiple different transcriptome assemblies. The script identifies the presence or absence of each of the proteins in each of the assemblies, thus providing an easy way to compare the gene repertoire of multiple different assemblies.

\section{Competing interests}

The authors declare that they have no competing interests.

\section{Authors' contributions}

DJS performed field collection of specimens and developmental manipulations, photography, RNA isolation, CDNA library synthesis. DJS and TJL performed divergence dating analysis. TJL and BRG assembled the reference transcriptome. BRG performed the saturation curve and metabolic pathway analysis. TJL and BRG carried out BLAST and GO analyses. TJL, ALB, and DJS performed gene family recovery. AMR and DJS carried out phylogenetic analyses. DJS, LD, AL, and JRF carried out MEME analyses. TJL and BRG constructed the database and the internet interface for Edwardsiellabase. DJS, TJL, BRG and AMR and JRF contributed to production of the figures and drafting of the manuscript. JRF oversaw the study design data analysis, production of figures, and the writing. All authors read and approved the final manuscript.

\section{Acknowledgements}

This research was supported by NSF grants MCB-0924749 and IOS-0818831 to JRF. DJS was supported by training grant NIH/NIGMS F31 GM095289-01 to JRF. Data generation and analysis were also supported by the budget of "Marine Genomics," a course in the Boston University Marine Semester. AMR received funding from the University of North Carolina at Charlotte. We are grateful to T. Gilmore for helpful comments on the manuscript. We would also like to thank Kevin Peterson for advice on the evolutionary divergence dating, and two annonymous reviewers, whose comments improved this manuscript.

\section{Author details}

'Department of Biology, Boston University, 5 Cummington Mall, Boston, MA 02215, USA. ${ }^{2}$ Bioinformatics Program, Boston University, 24 Cummington Mall, Boston, MA 02215, USA. ${ }^{3}$ Department of Biology, University of North Carolina at Charlotte, Charlotte, NC 28223, USA. ${ }^{4}$ Marine Program, Boston University, 5 Cummington Street, Boston, MA 02215, USA.

Received: 18 July 2013 Accepted: 11 December 2013 Published: 28 January 2014

\section{References}

1. Price PW: Evolutionary biology of parasites. Monogr Pop Biol 1980, 15:1-237.

2. Windsor DA: Most of the species on Earth are parasites. Int J Parasitol 1998, 28(12):1939-1941.
3. Howard RS, Lively CM: Parasitism, mutation accumulation and the maintenance of sex. Nature 1994, 367(6463):554-557.

4. Lively CM: Host-parasite coevolution and sex. Bioscience 1996, 46(2):107-114.

5. Morran LT, Schmidt OG, Gelarden IA, Parrish RC, Lively CM: Running with the Red Queen: host-parasite coevolution selects for biparental sex. Science 2011, 333(6039):216-218.

6. Schulenburg H, Kurtz J, Moret Y, Siva-Jothy MT: Introduction. Ecological immunology. Phil Trans Royal Soc London B 2009, 364(1513):3-14.

7. Lafferty K, Morris A: Altered behavior of parasitized killifish increases susceptibility to predation by bird final hosts. Ecology 1996, 77(5):1390-1397.

8. Coltman DW, Pilkington JG, Smith JA, Pemberton JM: Parasite-mediated selection against inbred Soay sheep in a free-living, island population. Evolution 1999, 53:1259-1267.

9. Moore SL, Wilson K: Parasites as a viability cost of sexual selection in natural populations of mammals. Science 2002, 297(5589):2015-2018.

10. Lafferty K: How environmental stress affects the impacts of parasites. Limnol Oceanog 1999, 44:925-931.

11. Smith KF, Sax DF, Lafferty KD: Evidence for the role of infectious disease in species extinction and endangerment. Cons Biol 2006, 20(5):1349-1357.

12. Poulin R: Evolutionary Ecology of Parasites. 2nd edition. Princeton, $\mathrm{NJ}$ : Princeton University Press; 2006.

13. Poulin R, Morand S: Parasite Biodiversity. Washington, D.C.: Smithsonian Institution Scholarly Press; 2004

14. Grbić M, Strand MR: Shifts in the life history of parasitic wasps correlate with pronounced alterations in early development. Proc Natl Acad Sci U S A 1998, 95(3):1097-1101.

15. Brooks DR: Lessons from a quiet classic. J Parasitol 2003, 89(5):878-885.

16. Crowell S: An edwardsiid larva parasitic in Mnemiopsis. In Coelenterate Ecology and Behavior. Edited by Mackie GO. New York: Plenum Press; 1976:247-250

17. Reitzel AM, Sullivan JC, Brown BK, Chin DW, Cira EK, Edquist SK, Genco BM, Joseph OC, Kaufman CA, Kovitvongsa K, et al: Ecological and developmental dynamics of a host-parasite system involving a sea anemone and two ctenophores. J Parasitol 2007, 93(6):1392-1402.

18. Selander E, Møller LF, Sundberg P, Tiselius P: Parasitic anemone infects the invasive ctenophore Mnemiopsis leidyi in the North East Atlantic. Biol Invasions 2010, 12:1003-1009.

19. Reitzel AM, Sullivan JC, Finnerty JR: Qualitative shift to indirect development in the parasitic sea anemone Edwardsiella lineata. Integr Comp Biol 2006, 46(6):827-837.

20. Reitzel A, Daly M, Sullivan J, Finnerty J: Comparative anatomy of developmental and parasitic stages in the life cycle of the lined sea anemone Edwardsiella lineata. J Parasitol 2009, 95(1):100-112.

21. Darling JA, Reitzel AR, Burton PM, Mazza ME, Ryan JF, Sullivan JC, Finnerty JR: Rising starlet: the starlet sea anemone. Nematostella vectensis. Bioessays 2005, 27(2):211-221.

22. Wolenski FS, Layden MJ, Martindale MQ, Gilmore TD, Finnerty JR: Characterizing the spatiotemporal expression of RNAs and proteins in the starlet sea anemone, Nematostella vectensis. Nat Protoc 2013, 8(5):900-915.

23. Stefanik DJ, Friedman LE, Finnerty JR: Collecting, rearing, spawning and inducing regeneration of the starlet sea anemone, Nematostella vectensis. Nat Protoc 2013, 8(5):916-923.

24. Stefanik DJ, Wolenski FS, Friedman LE, Gilmore TD, Finnerty JR: Isolation of DNA, RNA and protein from the starlet sea anemone Nematostella vectensis. Nat Protoc 2013, 8(5):892-899.

25. Daly M: A systematic revision of the Edwardsiidae (Cnidaria, Anthozoa). Invert Biol 2002, 2002(3):212-225.

26. Zerbino DR, Birney E: Velvet: algorithms for de novo short read assembly using de Bruijn graphs. Biotechfor 2008, 18(5):821-829.

27. Schulz MH, Zerbino DR, Vingron M, Birney E: Oases: robust de novo RNA-seq assembly across the dynamic range of expression levels. Bioinformatics 2012, 28(8):1086-1092.

28. Shinzato C, Shoguchi E, Kawashima T, Hamada M, Hisata K, Tanaka M, Fujie M, Fujiwara M, Koyanagi R, Ikuta T, et al: Using the Acropora digitifera genome to understand coral responses to environmental change. Nature 2011, 476(7360):320-323.

29. Park E, Hwang DS, Lee JS, Song JI, Seo TK, Won YJ: Estimation of divergence times in cnidarian evolution based on mitochondrial protein-coding genes and the fossil record. Mol Phylogenet Evol 2012, 62(1):329-345 
30. Pick KS, Philippe $H$, Schreiber F, Erpenbeck D, Jackson DJ, Wrede $P$, Wiens M, Alie A, Morgenstern B, Manuel M, et al: Improved phylogenomic taxon sampling noticeably affects nonbilaterian relationships. Mol Biol Evol 2010, 27(9):1983-1987.

31. Kerr AM: Molecular and morphological supertree of stony corals (Anthozoa: Scleractinia) using matrix representation parsimony. Biol Rev Camb Philos Soc 2005, 80(4):543-558.

32. Daly M, Fautin DG, Cappola VA: Systematics of the Hexcorallia (Cnidaria: Anthozoa). Zool J Linn Soc 2003, 139:419-437.

33. Daly M, Lipscomb DL, Allard MW: A simple test: evaluating explanations for the relative simplicity of the Edwardsiidae (Cnidaria: Anthozoa). Evolution 2002, 56(3):502-510.

34. Rodriguez E, Daly M: Phylogenetic relationships among deep-sea and chemosynthetic sea anemones: actinoscyphiidae and actinostolidae (Actiniaria: Mesomyaria). PloS One 2010, 5(6):e10958.

35. Daly M, Chaudhuri A, Gusmao L, Rodriguez E: Phylogenetic relationships among sea anemones (Cnidaria: Anthozoa: Actiniaria). Mol Phylogenet Evol 2008, 48(1):292-301.

36. Berntson EA, France SC, Mullineaux LS: Phylogenetic relationships within the class Anthozoa (phylum Cnidaria) based on nuclear 18S rDNA sequences. Mol Phylogenet Evol 1999, 13(2):417-433.

37. Peterson KJ, Lyons JB, Nowak KS, Takacs CM, Wargo MJ, McPeek MA: Estimating metazoan divergence times with a molecular clock. Proc Nat Acad Sci USA 2004, 101(17):6536-6541.

38. Rota-Stabelli O, Campbell L, Brinkmann H, Edgecombe GD, Longhorn SJ, Peterson KJ, Pisani D, Philippe H, Telford MJ: A congruent solution to arthropod phylogeny: phylogenomics, microRNAs and morphology support monophyletic Mandibulata. Proceedings Biological sciences/The Royal Society 2011, 278(1703):298-306.

39. Sperling EA, Robinson JM, Pisani D, Peterson KJ: Where's the glass? Biomarkers, molecular clocks, and microRNAs suggest a 200-Myr missing Precambrian fossil record of siliceous sponge spicules. Geobiology 2010, 8(1):24-36.

40. Maloof AC, et al: The earliest Cambrian record of animals and ocean geochemical change. Geol Soc Am Bull 2010, 122:1731.

41. Xingliang Zhang WL, Yuanlong Z: Cambrian Burgess Shale-type Lagerstatten in South China: distribution and significance. Gondwana Res 2008, 14(1-2):255-262

42. Peterson KJ, Cotton JA, Gehling JG, Pisani D: The Ediacaran emergence of bilaterians: congruence between the genetic and the geological fossil records. Philos T Roy Soc B 2008, 363(1496):1435-1443.

43. Paulyn Cartwright SLH, Hendricks JR, Jarrard RD, Marques AC, Collins AG, Lieberman BS: Exceptionally preserved jellyfishes from the Middle Cambrian. PloS one 2007, 2(10):e1121.

44. Benton MJ, Donoghue PC: Paleontological evidence to date the tree of life. Mol Biol Evol 2007, 24(1):26-53.

45. Xian-Guang Hou GS, Jie Z, Xiao-Ya M: Cambrian anemones with preserved soft tissue from the Chengjiang biota, China. Lethaia 2005, 38(3):193-203.

46. Peterson KJ, Butterfield NJ: Origin of the Eumetazoa: testing ecological predictions of molecular clocks against the Proterozoic fossil record. Proc Natl Acad Sci U S A 2005, 102(27):9547-9552.

47. Muller K, Walossek D, Zakharov A: Orsten' type phosphatized soft-integument preservation and a new record from the Middle Cambrian Kuonamka Formation in Siberia. N Jb Geol Paläontol Abh 1997, 197(101):1-118.

48. Erwin DH, Laflamme M, Tweedt SM, Sperling EA, Pisani D, Peterson KJ: The Cambrian conundrum: early divergence and later ecological success in the early history of animals. Science 2011, 334(6059):1091-1097.

49. Putnam NH, Srivastava M, Hellsten U, Dirks B, Chapman J, Salamov A, Terry A, Shapiro H, Lindquist E, Kapitonov W, et al: Sea anemone genome reveals ancestral eumetazoan gene repertoire and genomic organization. Science 2007, 317(5834):86-94.

50. Ogata H, Goto S, Sato K, Fujibuchi W, Bono H, Kanehisa M: KEGG: Kyoto Encyclopedia of Genes and Genomes. Nucleic acids research 1999, 27(1):29-34

51. Letunic I, Yamada T, Kanehisa M, Bork P: iPath: interactive exploration of biochemical pathways and networks. Trends Biochem Sci 2008 33(3):101-103.

52. Kusserow A, Pang K, Sturm C, Hrouda M, Lentfer J, Schmidt HA, Technau U, von Haeseler A, Hobmayer B, Martindale $M Q$, et al: Unexpected complexity of the Wnt gene family in a sea anemone. Nature 2005, 433(7022):156-160.
53. Srivastava M, Larroux C, Lu DR, Mohanty K, Chapman J, Degnan BM, Rokhsar DS: Early evolution of the LIM homeobox gene family. BMC Biol 2010, 8:4.

54. Ryan JF, Burton PM, Mazza ME, Kwong GK, Mullikin JC, Finnerty JR: The cnidarian-bilaterian ancestor possessed at least 56 homeoboxes: evidence from the starlet sea anemone, Nematostella vectensis. Genome Biol 2006, 7(7):R64.

55. Reitzel AM, Tarrant AM: Nuclear receptor complement of the cnidarian Nematostella vectensis: phylogenetic relationships and developmental expression patterns. BMC Evol Biol 2009, 9:230.

56. Zenkert C, Takahashi T, Diesner MO, Ozbek S: Morphological and molecular analysis of the Nematostella vectensis cnidom. PloS one 2011, 6(7):e22725.

57. Simionato E, Ledent V, Richards G, Thomas-Chollier M, Kerner P, Coornaert D, Degnan BM, Vervoort M: Origin and diversification of the basic helix-loop-helix gene family in metazoans: insights from comparative genomics. BMC Evol Biol 2007, 7:33.

58. Reitzel AM, Sullivan JC, Finnerty JR: Discovering SNPs in protein coding regions with StellaSNP: Illustrating the characterization and geographic distribution of polymorphisms in the estuarine anemone Nematostella vectensis. Estuar Coast 2010, 33(4):930-943.

59. Magie CR, Pang K, Martindale MQ: Genomic inventory and expression of Sox and Fox genes in the cnidarian Nematostella vectensis. Dev Genes Evol 2005, 215(12):618-630.

60. Sullivan JC, Wolenski FS, Reitzel AM, French CE, Traylor-Knowles N, Gilmore TD, Finnerty JR: Two alleles of NF-kappaB in the sea anemone Nematostella vectensis are widely dispersed in nature and encode proteins with distinct activities. PloS one 2009, 4(10):e7311.

61. Wolenski FS, Bradham CA, Finnerty JR, Gilmore TD: NF-kappaB is required for cnidocyte development in the sea anemone Nematostella vectensis. Dev Biol 2013, 373(1):205-215.

62. Wolenski FS, Chandani S, Stefanik DJ, Jiang N, Chu E, Finnerty JR, Gilmore TD: Two polymorphic residues account for the differences in DNA binding and transcriptional activation by NF-kappaB proteins encoded by naturally occurring alleles in Nematostella vectensis. J Mol Evol 2011, 73(5-6):325-336.

63. Wolenski FS, Garbati MR, Lubinski TJ, Traylor-Knowles N, Dresselhaus E, Stefanik DJ, Goucher H, Finnerty JR, Gilmore TD: Characterization of the core elements of the NF-kappaB signaling pathway of the sea anemone Nematostella vectensis. Mol Cell Bio/ 2011, 31(5):1076-1087.

64. Sullivan JC, Kalaitzidis D, Gilmore TD, Finnerty JR: Rel homology domain-containing transcription factors in the cnidarian Nematostella vectensis. Dev Genes Evol 2007, 217(1):63-72.

65. Gilmore TD, Wolenski FS: NF-kappaB: where did it come from and why? Immunol Rev 2012, 246(1):14-35.

66. Sullivan JC, Ryan JF, Mullikin JC, Finnerty JR: Conserved and novel Wnt clusters in the basal eumetazoan Nematostella vectensis. Dev Genes Evol 2007, 217(3):235-239.

67. Traylor-Knowles N, Granger BR, Lubinski T, Parikh JR, Garamszegi S, Xia Y, Marto JA, Kaufman L, Finnerty JR: Production of a reference transcriptome and a transcriptomic database (PocilloporaBase) for the cauliflower coral, Pocillopora damicornis. BMC Genomics 2011, 12(1):585.

68. Langmead B, Salzberg SL: Fast gapped-read alignment with Bowtie 2. Nat Methods 2012, 9(4):357-359.

69. Skinner ME, Uzilov AV, Stein LD, Mungall CJ, Holmes IH: JBrowse: a next-generation genome browser. Biotechfor 2009, 19(9):1630-1638.

70. Westesson $\mathrm{O}$, Skinner $\mathrm{M}$, Holmes I: Visualizing next-generation sequencing data with JBrowse. Brief Bioinform 2013, 14(2):172-177.

71. Krishna S, Nair A, Cheedipudi S, Poduval D, Dhawan J, Palakodeti D, Ghanekar Y: Deep sequencing reveals unique small RNA repertoire that is regulated during head regeneration in Hydra magnipapillata. Nucleic Acids Res 2013, 41(1):599-616.

72. Lehnert EM, Burriesci MS, Pringle JR: Developing the anemone Aiptasia as a tractable model for cnidarian-dinoflagellate symbiosis: the transcriptome of aposymbiotic A. pallida. BMC Genomics 2012, 13:271

73. Meyer E, Aglyamova GV, Wang S, Buchanan-Carter J, Abrego D, Colbourne JK, Willis BL, Matz MV: Sequencing and de novo analysis of a coral larval transcriptome using 454 GSFlx. BMC Genomics 2009, 10:219.

74. Moya A, Huisman L, Ball EE, Hayward DC, Grasso LC, Chua CM, Woo HN, Gattuso JP, Foret S, Miller DJ: Whole transcriptome analysis of the coral Acropora millepora reveals complex responses to $\mathrm{CO}(2)$-driven acidification during the initiation of calcification. Mol Ecol 2012, 21(10):2440-2454 
75. Polato NR, Vera JC, Baums IB: Gene discovery in the threatened elkhorn coral: 454 sequencing of the Acropora palmata transcriptome. PloS one 2011, 6(12):e28634.

76. Portune KJ, Voolstra CR, Medina M, Szmant AM: Development and heat stress-induced transcriptomic changes during embryogenesis of the scleractinian coral Acropora palmata. Mar Genom 2010, 3(1):51-62.

77. Sabourault C, Ganot P, Deleury E, Allemand D, Furla P: Comprehensive EST analysis of the symbiotic sea anemone, Anemonia viridis. BMC Genomics 2009, 10:333.

78. Soza-Ried J, Hotz-Wagenblatt A, Glatting KH, del Val C, Fellenberg K, Bode HR, Frank U, Hoheisel JD, Frohme M: The transcriptome of the colonial marine hydroid Hydractinia echinata. FEBS J 2010, 277(1):197-209.

79. Sun J, Chen Q, Lun JCY, Xu JL, Qiu JW: PcarnBase: development of a transcriptomic database for the brain coral platygyra carnosus. Mar Biotechnol 2013, 15(2):244-251.

80. Sunagawa S, Wilson EC, Thaler M, Smith ML, Caruso C, Pringle JR, Weis VM, Medina M, Schwarz JA: Generation and analysis of transcriptomic resources for a model system on the rise: the sea anemone Aiptasia pallida and its dinoflagellate endosymbiont. BMC Genomics 2009, 10:258

81. Siebert S, Robinson MD, Tintori SC, Goetz F, Helm RR, Smith SA, Shaner N, Haddock SH, Dunn CW: Differential gene expression in the siphonophore Nanomia bijuga (Cnidaria) assessed with multiple next-generation sequencing workflows. PloS one 2011, 6(7):e22953.

82. Vize PD: Transcriptome analysis of the circadian regulatory network in the coral Acropora millepora. Biol Bull 2009, 216(2):131-137.

83. Voolstra CR, Schwarz JA, Schnetzer J, Sunagawa S, Desalvo MK, Szmant AM, Coffroth MA, Medina M: The host transcriptome remains unaltered during the establishment of coral-algal symbioses. Mol Ecol 2009, 18(9):1823-1833.

84. Helm RR, Siebert S, Tulin S, Smith J, Dunn CW: Characterization of differential transcript abundance through time during Nematostella vectensis development. BMC Genomics 2013, 14:266

85. Tulin S, Aguiar D, Istrail S, Smith J: A quantitative reference transcriptome for Nematostella vectensis early embryonic development: a pipeline for de novo assembly in emerging model systems. Evo Devo 2013, 4(1):16.

86. Francis WR, Christianson LM, Kiko R, Powers ML, Shaner NC, D Haddock SH: A comparison across non-model animals suggests an optimal sequencing depth for de novo transcriptome assembly. BMC Genomics 2013, 14:167.

87. Daly M: Taxonomy, anatomy, and histology of the lined sea anemone, Edwardsiella lineata (Verrill, 1873) (Cnidaria: Anthozoa: Edwardsiidae). Proc Biol Soc Wash 2002, 115(4):868-877.

88. Hinman VF, Nguyen AT, Cameron RA, Davidson EH: Developmental gene regulatory network architecture across 500 million years of echinoderm evolution. Proc Natl Acad Sci USA 2003, 100(23):13356-13361.

89. Romano LA, Wray GA: Conservation of Endo16 expression in sea urchins despite evolutionary divergence in both cis and trans-acting components of transcriptional regulation. Development 2003, 130(17):4187-4199.

90. Bu D, Yu K, Sun S, Xie C, Skogerbo G, Miao R, Xiao H, Liao Q, Luo H, Zhao G, et al: NONCODE v3.0: integrative annotation of long noncoding RNAs. Nucleic Acids Res 2012, 40(Database issue):D210-D215.

91. Tsai IJ, Zarowiecki M, Holroyd N, Garciarrubio A, Sanchez-Flores A, Brooks KL, Tracey A, Bobes RJ, Fragoso G, Sciutto E, et al: The genomes of four tapeworm species reveal adaptations to parasitism. Nature 2013, 496(7443):57-63.

92. Sullivan JC, Reitzel AM, Finnerty JR: Upgrades to StellaBase facilitate medical and genetic studies on the starlet sea anemone, Nematostella vectensis. Nucleic Acids Res 2008, 36(Database issue):D607-D611.

93. Sullivan JC, Ryan JF, Watson JA, Webb J, Mullikin JC, Rokhsar D, Finnerty JR: StellaBase: the Nematostella vectensis Genomics Database. Nucleic Acids Res 2006, 34(Database issue):D495-D499.

94. Ronquist F, Huelsenbeck JP: MrBayes 3: Bayesian phylogenetic inference under mixed models. Bioinformatics 2003, 19(12):1572-1574.

95. Lartillot N, Lepage T, Blanquart S: PhyloBayes 3: a Bayesian software package for phylogenetic reconstruction and molecular dating Bioinformatics 2009, 25(17):2286-2288.

96. Lartillot N, Rodrigue N, Stubbs D, Richer J: PhyloBayes MPI: phylogenetic reconstruction with infinite mixtures of profiles in a parallel environment. Syst Biol 2013, 62(4):611-615.

97. Edgar RC: MUSCLE: multiple sequence alignment with high accuracy and high throughput. Nucleic Acids Res 2004, 32(5):1792-1797.

98. Abascal F, Zardoya R, Posada D: ProtTest: selection of best-fit models of protein evolution. Bioinformatics 2005, 21(9):2104-2105.
99. Stamatakis A: RAxML-VI-HPC: maximum likelihood-based phylogenetic analyses with thousands of taxa and mixed models. Bioinformatics 2006, 22(21):2688-2690.

100. The CIPRES Science Gateway: a community resource for phylogenetic analysis: http://www.phylo.org/index.php/portal/.

101. Stamatakis $A$, Hoover $P$, Rougemont J: A rapid bootstrap algorithm for the RAxML Web servers. Syst Biol 2008, 57(5):758-771.

102. Castresana J: Selection of conserved blocks from multiple alignments for their use in phylogenetic analysis. Mol Biol Evol 2000, 17:540-552.

103. Bailey TL: Discovering novel sequence motifs with MEME. Curr Protoc Bioinformatics 2002, Chapter 2:Unit 24.

doi:10.1186/1471-2164-15-71

Cite this article as: Stefanik et al:: Production of a reference

transcriptome and transcriptomic database (EdwardsiellaBase) for the lined sea anemone, Edwardsiella lineata, a parasitic cnidarian. BMC Genomics 2014 15:71.

\section{Submit your next manuscript to BioMed Central and take full advantage of:}

- Convenient online submission

- Thorough peer review

- No space constraints or color figure charges

- Immediate publication on acceptance

- Inclusion in PubMed, CAS, Scopus and Google Scholar

- Research which is freely available for redistribution 